\title{
Gas stripping in galaxy clusters: a new SPH simulation approach ${ }^{\star}$
}

\author{
P. Jáchym ${ }^{1}$, J. Palouš ${ }^{1}$, J. Köppen ${ }^{1,2,3,4}$, and F. Combes ${ }^{5}$ \\ 1 Astronomical Institute, Academy of Sciences of the Czech Republic, Boční II 1401, 14131 Prague 4, Czech Republic \\ e-mail: [jachym;palous]@ig.cas.cz \\ 2 Observatoire Astronomique de Strasbourg, 11 rue de l'Université, 67000 Strasbourg, France \\ e-mail: koppen@astro.u-strasbg.fr \\ 3 International Space University, Parc d'Innovation, 1 rue Jean-Dominique Cassini, 67400 Illkirch-Graffenstaden, France \\ 4 Institut für Theoretische Physik und Astrophysik, Universität Kiel, 24098 Kiel, Germany \\ 5 Observatoire de Paris, LERMA, 61 Av. de l'Observatoire, 75014 Paris, France
}

Received 25 September 2006 / Accepted 22 May 2007

\section{ABSTRACT}

\begin{abstract}
Aims. The influence of a time-varying ram pressure on spiral galaxies in clusters is explored with a new simulation method based on the $N$-body SPH/tree code GADGET.

Methods. We have adapted the code to describe the interaction of two different gas phases, the diffuse hot intracluster medium (ICM) and the denser and colder interstellar medium (ISM). Both the ICM and ISM components are introduced as SPH particles. As a galaxy arrives on a highly radial orbit from outskirts to cluster center, it crosses the ICM density peak and experiences a time-varying wind. Results. Depending on the duration and intensity of the ISM-ICM interaction, early and late type galaxies in galaxy clusters with either a large or small ICM distribution are found to show different stripping efficiencies, amounts of reaccretion of the extra-planar ISM, and final masses. We compare the numerical results with analytical approximations of different complexity and indicate the limits of the Gunn \& Gott simple stripping formula.

Conclusions. Our investigations emphasize the role of the galactic orbital history to the stripping amount. We discuss the contribution of ram pressure stripping to the origin of the ICM and its metallicity. We propose gas accumulations like tails, filaments, or ripples to be responsible for stripping in regions with low overall ICM occurrence.
\end{abstract}

Key words. galaxies: interactions - galaxies: intergalactic medium - galaxies: clusters: general - ISM: kinematics and dynamics hydrodynamics - methods: $N$-body simulations

\section{Introduction}

Galaxies in clusters and rich environments are observed to be stripped of their interstellar medium, which quenches subsequent star formation. The stripping can be due either to tidal interactions, spiral galaxy mergers into ellipticals, or to rampressure stripping from the intracluster gas. Both tidal and rampressure stripping are always tightly linked. Gunn \& Gott (1972) assume that, after the formation of a galaxy cluster, the remaining gaseous debris is thermalized via shock heating to virial temperatures corresponding to random motions in the cluster, e.g. to a few times $10^{7} \mathrm{~K}$. This hot plasma at densities of a few times $10^{-3} \mathrm{~cm}^{-3}$ influences the ISM in disks of spiral galaxies and can remove part of their gas through the ram pressure induces by the galaxy motions through the ICM.

The ram pressure stripping is difficult to model, since several complex gaseous phenomena are involved. The first aspect is the simple pressure force, and this can be modeled with a simple algorithm using ballistic and sticky particles to represent the gas (e.g. Vollmer et al. 2001). But the full hydrodynamical processes include thermal evaporation, turbulent and viscous stripping, and also outflows due to star formation.

The first notice of the 3D $N$-body/SPH simulations with the gravity tree to mimic the dynamical effect of the ram pressure on galaxies in clusters is by Kundic et al. (1993). The same approach has been adopted by Abadi et al. (1999). The ICM is

* Appendix A is only available in electronic form at http: //www . aanda. org represented as a flow of particles along a cylinder of radius $30 \mathrm{kpc}$ and thickness $10 \mathrm{kpc}$. A spiral galaxy is in face-on, edgeon, or an inclined orientation relative to flow of ICM particles. With the simulations they examine the radius up to which the ISM is removed and compare the results with the prediction of Gunn \& Gott (1972) that the ISM is removed from the disk if the ICM ram pressure exceeds the restoring force:

$\rho_{\mathrm{ICM}} v^{2} \geq\left.\frac{\partial \Phi(r, z)}{\partial z}\right|_{\max } \Sigma_{\mathrm{ISM}}$,

where $\rho_{\text {ICM }}$ is the ICM density, $v$ the relative velocity of the galaxy and the ICM, $\Phi(r, z)$ the total gravitational potential of the galaxy as a function of the galactocentric cylindrical coordinates, and $\Sigma_{\text {ISM }}$ the ISM surface density. The conclusion is that Eq. (1) applies in the face-on galaxy orientation when the bulge does not dominate the disk gravity. In other cases, edge-on orientation and in central parts of galaxies, where bulge dominates, the stripping is less efficient. The time scale for gas removal is $\sim 10^{8}$ years.

The ISM is far from continuous, but instead consists of several components: hot, warm, and cold medium. Hot ionized and warm neutral H I medium are much more continuous compared to cloudy, cold, and molecular component. The SPH description relates more to the continuous ISM components, while molecular clouds are not represented well in the SPH simulations. Vollmer et al. (2001) adopt another approach, using $N$-body simulations with sticky particles representing the inelastic collisions between molecular clouds. The ram pressure is included with an additional friction force acting on clouds in the wind direction. 
Vollmer et al. (2001, 2006) introduce a time dependent ram pressure, which corresponds to the variations of the ICM density and of relative ICM-ISM velocity along the galaxy orbit in the cluster. They allow a moving ICM, which may increase its velocity relative the ISM to values higher than $4000 \mathrm{~km} \mathrm{~s}^{-1}$.

With an isothermal SPH gas model, Abadi et al. (1999) show that gas stripping can be quite efficient in the core of rich clusters on a time-scale of $10^{7} \mathrm{yrs}$. The most efficient is face-on orientation. With a Eulerian code, Quilis et al. (2000) probed the efficiency of viscous coupling, enhanced by the presence of $\mathrm{H} \mathrm{I}$ deficiencies in the center of galaxies. The ram pressure stripping does not strongly depend on the vertical structure and thickness of the gas disk (Roediger \& Hensler 2005). In more detail, the effect of inclination of the moving galaxy is important as long as the ram pressure is comparable to the gas pressure in the galaxy plane. In general the effect is similar for all inclinations except for edge-on (Roediger \& Brüggen 2006). The orientation of the gas tail behind the galaxy is not a good tracer of the galaxy motion on its orbit. Acreman et al. (2003) simulate the infall of an elliptical galaxy and its gas stripping by the ICM showing that the formation of an X-ray wake at the first passage should be observable.

The principal competing mechanism for perturbing galaxies in clusters and stripping their gas is tidal interactions. Highspeed galaxy encounters in clusters, galaxy harassment events, are efficient mechanisms for disturbing galaxy disks, and providing stars to the intracluster space (Moore et al. 1996). Also low-speed galaxy encounters in groups before the formation of the cluster are able to drag tidal stellar tails out and lead to galaxy mergers and formation of ellipticals, which are then known to be more numerous in clusters. The evolution of encounter debris in galaxy haloes has been followed by Mihos (2004).

Intracluster light is a consequence of the tidal stripping of galaxies. It has been determined that intra-cluster stars are older than galaxy stellar populations and more centrally concentrated in the cluster. The fraction of stars in the ICM is increasing with the richness of the cluster and it is usually a few percent of all stars, up to $20 \%$ (Arnaboldi et al. 2003; Murante et al. 2004). If the ICM metallicity can be explained partly by gas stripping from galaxies (Domainko et al. 2006), it can also come from intra-cluster supernovae exploding in the ICM. The irregular structures in galaxy clusters found in X-ray are tracing cold fronts and shocks due to galaxy interactions or to the infall of a galaxy group.

The fate of the stripped ISM in clusters is not clear, because it can end very hot and become part of the ICM or stay quite cold condensing into clouds with a molecular core and an H I outer boundary. Oosterloo \& van Gorkom (2005) observed a large H I cloud near the center of the Virgo cluster and suggest that this cloud has been ram-pressure stripped from the galaxy NGC 4388. The dense clumps in the H I plume might be molecular, and star formation could occur precisely at these high-density places.

In this paper, we focus on computations of the ram pressure stripping, to evaluate its efficiency more precisely and to follow the fate of the stripped gas. The particular goal is to take the finite time for this stripping interaction into account, as the galaxy passes quickly through the central region of the cluster. Most computations until now were carried out in the hypothesis of a stationary wind of hot gas. But the ram pressure acts only in a short lapse of time, as simulated by Vollmer et al. (2001). After this short period, a certain fraction of the gas falls back onto the galaxy. These latter simulations did not take into account both the pressure forces and the hydrodynamical physics of the
ISM-ICM interactions. Roediger et al. (2006) have followed the wake of gas produced by a quasi-stationary wind; here, on the contrary, we emphasize the impulsive character of ram pressure stripping.

Assuming a static ICM described with a $\beta$-profile, we explore the ISM-ICM interaction in detail during the galaxy crossing of the central part of the cluster. The mass-loss rates are examined as a function of the galaxy type, size, and mass. We are able to follow the gas stripped from the galaxy, forming a giant tail of material. We tackle the issue of the origin of the ICM and the actual role of gas stripping in building it as a function of the cluster mass and richness. The importance of gas stripping could account for the metal enrichment of the ICM as a function of cluster type (e.g. Domainko et al. 2006; Schindler et al. 2005). After presenting the model of stripped galaxies and of the cluster in Sects. 2 and 3, we introduce the initial conditions in Sect. 4 and describe our simulation method in Sect. 5. In Sect. 6, we discuss the results of the numerical simulations, the simulation test is shown in Sect. 7, and the equation of motion of an individual ISM gas element and the impulse approximation are given and applied to stripping in Sect. 8. The present simulations are compared to other simulations in Sect. 9. Section 10 presents the discussion and conclusion.

\section{The galaxy model}

Our model of a spiral galaxy is a standard three-component halo + bulge + disk configuration. The halo and bulge components are given with spherically symmetric, Plummer density distributions

$\rho_{\mathrm{b}, \mathrm{h}}(d)=\rho_{0} \frac{a_{\mathrm{b}, \mathrm{h}}^{5}}{\left(d^{2}+a_{\mathrm{b}, \mathrm{h}}^{2}\right)^{5 / 2}}$,

where $\rho_{0}=3 M_{\mathrm{b}, \mathrm{h}} / 4 \pi a_{\mathrm{b}, \mathrm{h}}^{3}$ is the central density, $d$ the distance from the galactic center, $M_{\mathrm{b}}, M_{\mathrm{h}}$ are total masses, and $a_{\mathrm{b}}$ and $a_{\mathrm{h}}$ are the radial scaling factors corresponding to the bulge or halo.

The axially symmetric disk follows an infinitely thin Toomre-Kuzmin disk multiplied by a $\operatorname{sech}^{2}\left(z / z_{0}\right)$ term that defines its isothermal vertical profile,

$\rho_{\mathrm{d}}(r, z)=\rho_{0} \frac{a_{\mathrm{d}}^{3}}{\left(r^{2}+a_{\mathrm{d}}^{2}\right)^{3 / 2}} \operatorname{sech}^{2}\left(z / z_{0}\right)$,

where $(r, z)$ are the galactocentric cylindrical coordinates, and $a_{\mathrm{d}}$ and $z_{0}$ are the disk scaling factors. The central density is determined by $\rho_{0}=M_{\mathrm{d}} / 4 \pi a_{\mathrm{d}}^{2} z_{0}$ with the total mass $M_{\mathrm{d}}$ of the disk.

We introduce model spiral galaxies of various types: latetype (L) or early-type (E), subdivided according to their total mass: massive (M) or low-mass (m). The L-types have very low bulge-to-disk mass ratios compared to E-types, and M-types have a massive halo component compared to m-types. Thus we employ four models: LM, Lm, EM, and Em. The values of the model parameters are given in Table 1 . The scale height of the disk in all models is $z_{0}=0.25 \mathrm{kpc}$.

Our standard galaxy model is the LM-type. Its rotation curve, the escape velocity, and the gravitational restoring force $f_{\text {rest }}=$ $\Sigma_{\text {ISM }} \times \partial \Phi(r, z) / \partial z$ are shown in Fig. 1 , where $\Phi(r, z)$ is the total gravitational potential including all components of the galaxy, and $\partial \Phi / \partial z$ the total force attracting ISM elements towards the galaxy's symmetry plane. The escape velocity is obtained as a function $(r, z)$ from the potential: $v_{\mathrm{esc}}(r, z)=\sqrt{2|\Phi(r, z)|}$, and its maximum is always in the symmetry plane $z=0$. 

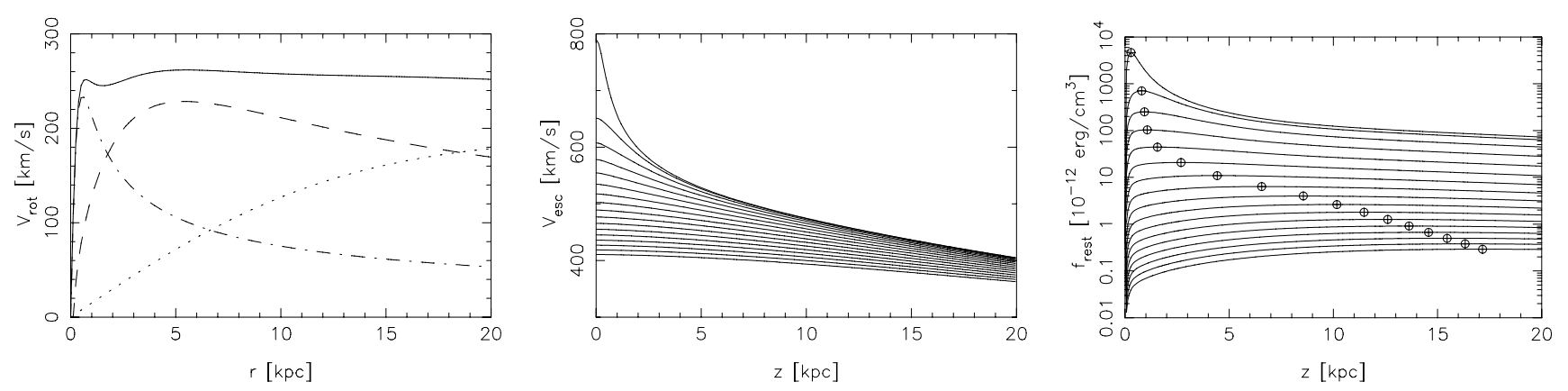

Fig. 1. LM model galaxy. Left: rotation curve with contributions of the halo (dotted), bulge (dash-dotted), and disk (dashed) components. Center: escape velocity $v_{\text {esc }}$ as a function of the $z$-distance behind the disk plane for 16 radii $r$ starting with $r=1 \mathrm{kpc}$ (top curve) and a spacing of $1 \mathrm{kpc}$. Right: gravitational restoring force $f_{\text {rest }}$ as a function of $z$ for 16 values of radius $r$ from $1 \mathrm{kpc}$ (top curve) to $16 \mathrm{kpc}$. Maxima in $z$ for individual radii are denoted with circles.
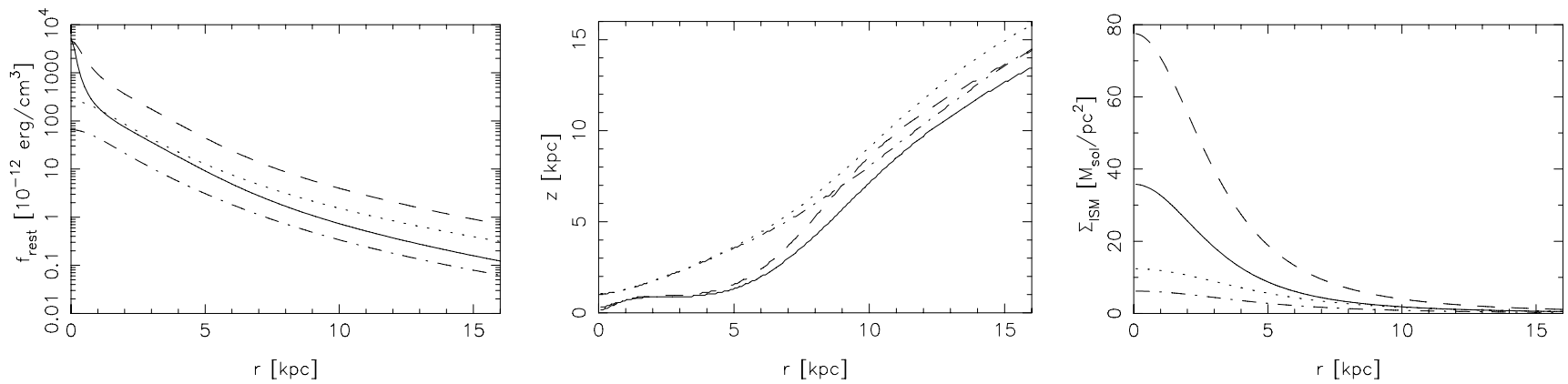

Fig. 2. Left: maximum restoring force over $z$ as a function of the galactocentric distance $r$ for the four galaxy models adopted: LM (dashed), Lm (full), EM (dotted), and Em (dash-dotted). Center: positions of the maximum values of the restoring force behind the disk plane for different radii $r$. Right: surface density $\Sigma_{\text {ISM }}$ of the ISM as a function of the galactocentric distance $r$.

Table 1. The disk, bulge, and halo parameters for late (L) or early (E), massive (M) or low-mass (m) type models of a spiral galaxy.

\begin{tabular}{cclcccc}
\hline \hline & & & LM & Lm & EM & Em \\
\hline disk: & $M_{\mathrm{d}}$ & $\left(10^{10} M_{\odot}\right)$ & 8.6 & 4 & 3.1 & 1.5 \\
& $a_{\mathrm{d}}$ & $(\mathrm{kpc})$ & 4 & 4 & 6 & 6 \\
bulge: & $M_{\mathrm{b}}$ & $\left(10^{10} M_{\odot}\right)$ & 1.3 & 0.5 & 7.3 & 3.5 \\
& $a_{\mathrm{b}}$ & $(\mathrm{kpc})$ & 0.5 & 0.1 & 1 & 1 \\
halo: & $M_{\mathrm{h}}$ & $\left(10^{10} M_{\odot}\right)$ & 42 & 14 & 49 & 16.4 \\
& $a_{\mathrm{h}}$ & $(\mathrm{kpc})$ & 20 & 20 & 25 & 25 \\
\hline
\end{tabular}

Rotation velocities of other three models, Lm, EM, and Em, are about $160 \mathrm{~km} \mathrm{~s}^{-1}, 220 \mathrm{~km} \mathrm{~s}^{-1}$, and $150 \mathrm{~km} \mathrm{~s}^{-1}$, respectively.

At a given $r$, the restoring force has a maximum at some distance $z$ from the symmetry plane. With increasing radius $r$, this maximum is farther and farther from the $z=0$ plane and its values decrease. In Fig. 2 (left panel) we plot the maxima of the restoring force as a function of $r$ for different galaxy types. The LM-type galaxy has a massive disk and massive halo, which gives the highest restoring force compared to other galaxy types. Both the disk and halo are less massive for the Lm-type, consequently, the restoring force is smaller; only in the central part, where the very concentrated bulge dominates, the restoring force increases rapidly. The bulge is much more massive and extended in an EM-type galaxy, so the restoring force in the outer galaxy parts overcomes that of an Lm-type. For radii between 1 and $4 \mathrm{kpc}$, galaxy models Lm and EM show very similar runs of the maxima of the restoring force with $r$, but the maxima occur at lower $z$ in the Lm-type (see Fig. 2, central panel). The Em-type galaxy, where the disk and halo have lower masses compared to the other types, has the weakest restoring force.
We assume that the ISM initially follows the density distribution of Eq. (3) and that it amounts to $10 \%$ of the total disk mass. The ISM surface density $\Sigma_{\text {ISM }}$ is plotted as a function of the galactocentric distance for the four galaxy types in the right hand panel of Fig. 2 .

\section{The model of a galaxy cluster}

The hot ICM was observed with X-ray satellites ROSAT and ASCA and later resolved spatially with satellites XMM-Newton and Chandra (Böhringer 2004). The large-scale ICM distribution in clusters is described with a $\beta$-profile (Cavaliere \& Fusco-Femiano 1976; Schindler et al. 1999):

$\rho_{\mathrm{ICM}}=\rho_{0, \mathrm{ICM}}\left(1+\frac{R^{2}}{R_{\mathrm{c}, \mathrm{ICM}}^{2}}\right)^{-\frac{3}{2} \beta_{\mathrm{ICM}}}$,

where $\rho_{\text {ICM }}$ denotes the ICM volume density at distance $R$ from the cluster center, $\rho_{0, \mathrm{ICM}}$ is the volume density of the ICM in the cluster center, $R_{\mathrm{c}, \mathrm{ICM}}$ a parameter of the ICM central concentration, and $\beta_{\mathrm{ICM}}$ the slope parameter, which we set according to Schindler et al. (1999) to $1 / 2$. Our standard cluster model has $\rho_{0, \mathrm{ICM}}=4 \times 10^{-3} \mathrm{~cm}^{-3}$ and $R_{\mathrm{c}, \mathrm{ICM}}=13.4 \mathrm{kpc}$. Vollmer et al. (2001) use a ten-times higher value of $\rho_{0, \text { ICM }}$ to represent the ICM distribution in the Virgo cluster. We discuss the difference in $\rho_{0, \mathrm{ICM}}$ setting in Sect. 9.3.

To model either a rich galaxy cluster with a lot of hot ICM or a poor cluster with just a little ICM in the center, we vary the values of $R_{\mathrm{C}, \mathrm{ICM}}$ and $\rho_{0, \mathrm{ICM}}$. We multiply either standard value with factors of $0.25,0.5,1,2$, and 4 . The left panel of Fig. 3 illustrates the effect of varying $R_{\mathrm{c}, \mathrm{ICM}}$ while $\rho_{0, \mathrm{ICM}}$ stays fixed. The width of the ICM density peak grows with increasing $R_{\mathrm{c}, \mathrm{ICM}}$. 

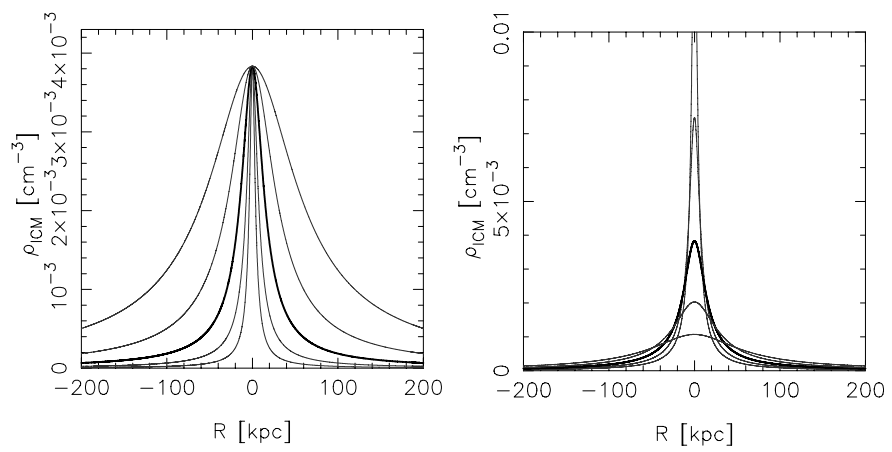

Fig. 3. Left: central view of the ICM density distribution in clusters with $R_{\mathrm{c}, \mathrm{ICM}}$ equal to $0.25,0.5,1,2,4 \times 13.4 \mathrm{kpc}$, and $\rho_{0, \mathrm{ICM}}=4 \times 10^{-3} \mathrm{~cm}^{-3}$. Right: peaks of the ICM density distribution with the following combinations of the $\beta$-profile parameters $\left(R_{\mathrm{c} \text { ICM }}, \rho_{0 \text { ICM }}\right):(0.25,3.82),(0.5$, $1.95),(1,1),(2,0.53)$, and $(4,0.28) \times\left(13.4 \mathrm{kpc}, 4 \times 10^{-3} \mathrm{~cm}^{-3}\right)$. A galaxy traversing such clusters on a completely radial orbit from $1 \mathrm{Mpc}$ distance crosses the same amount of the ICM.

When the galaxy flies through the cluster, it encounters a certain amount of ICM. The right panel of Fig. 3 corresponds to five clusters with combinations of $R_{\mathrm{c}, \mathrm{ICM}}, \rho_{0, \mathrm{ICM}}$ that make the galaxy cross the same amount of ICM when moving on a completely radial orbit, starting from a distance of $1 \mathrm{Mpc}$. The shape of the peak changes from narrow and high to wide and low.

The gravitational potential of a galaxy cluster is produced by the distributions of ICM and dark matter (DM) in the cluster. The DM is introduced through another $\beta$-profile. We set $R_{\mathrm{c}, \mathrm{DM}}=$ $320 \mathrm{kpc}, \rho_{0, \mathrm{DM}}=1.6 \times 10^{-2} \mathrm{~cm}^{-3}$, and $\beta_{\mathrm{DM}}=1$ as in Vollmer et al. (2001). The different steepness of gas and galaxy density profiles $\left(\beta_{\mathrm{ICM}}\right.$ versus $\left.\beta_{\mathrm{DM}}\right)$ corresponds closely to observations, with the ICM density profile steeper than the galaxy distribution in the inner part of the subclump. This implies DM of either $3.7 \times 10^{12} M_{\odot}$ or $1.4 \times 10^{14} M_{\odot}$ within $140 \mathrm{kpc}$ (see later) or $1 \mathrm{Mpc}$ from the cluster center. Correspondingly, depending on the type of the cluster, ICM represents from $0.05 \%$ to $30 \%$ of the mass within the central $140 \mathrm{kpc}$ (see Table 3).

\section{Initial conditions}

The three components of the model galaxy are represented by a number of particles of equal mass: 12000 for the halo, 6000 for the bulge, and 12000 for the stellar disk. Their initial distributions follow the density profiles of Eqs. (2) and (3). The components are cut off at $40 \mathrm{kpc}, 4 \mathrm{kpc}$, and $16 \mathrm{kpc}$, in the case of the halo, bulge, and disk, respectively. The ISM is represented with 12000 SPH particles of total mass equal to $10 \%$ of the total disk mass. This means that the mass of an ISM particle is in the range of $(1.5-5.7) \times 10^{5} M_{\odot}$ in the four introduced galaxy types. Their initial distribution follows the density profile of the stellar disk (Eq. (3); see right panel of Fig. 2).

The ICM of the cluster is modeled with 120000 SPH particles following the $\beta$-profile (Eq. (4)) with a cut-off at $R=$ $140 \mathrm{kpc}$ from the center of the cluster. The total mass of the ICM particles is derived from cluster parameters. In the case of the standard cluster, there is $6 \times 10^{10} M_{\odot}$ of the ICM within the central $140 \mathrm{kpc}$. Consequently the mass of an individual ICM particle is $\sim 5 \times 10^{5} M_{\odot}$, which is comparable to the mass of an ISM particle. For the LM-type galaxy and different clusters, the mass ratio of ISM to ICM particles ranges between 30 and 0.04 .

The cut-off radius of the ICM particles distribution set to $140 \mathrm{kpc}$ corresponds in the case of the standard model to the
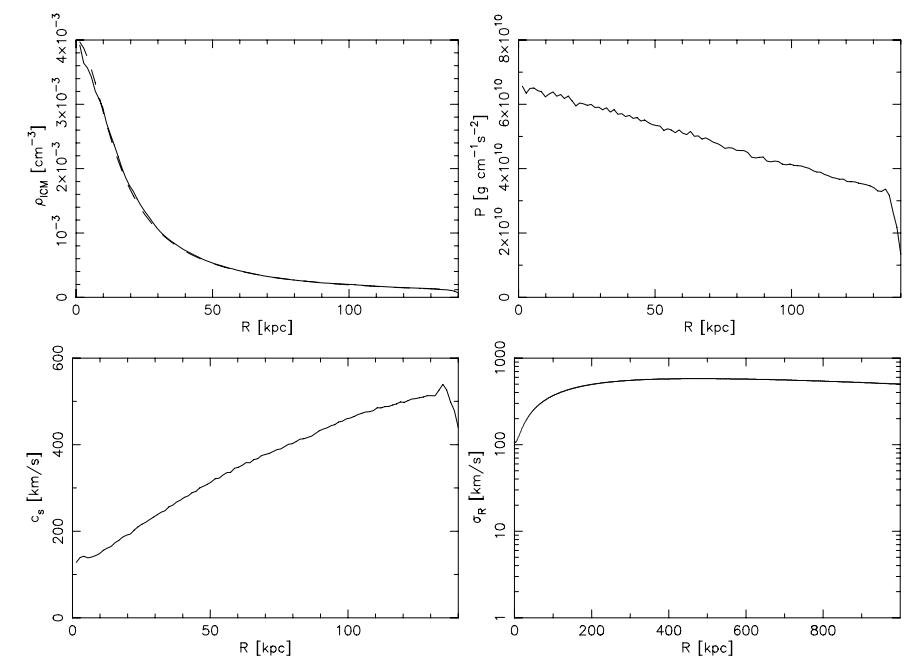

Fig. 4. Radial profiles of the ICM density, pressure, and speed of sound about $0.5 \mathrm{Gyr}$ before the galaxy enters the ICM distribution. The dashed curve in the density plot corresponds to Eq. (4). Lower right: initial profile of the ICM velocity dispersion $\sigma_{R}$, Eq. (5).

radius at which outer parts of the galaxy arriving from the cluster periphery start to be affected by steeply rising ram pressure of the crossed ICM. It means that we restrict the active sphere of the ICM distribution to central regions of the cluster, where the effect of the ICM-ISM interaction is strongest. At larger distances only the gravitational effect of the ICM to the galaxy is considered.

A more realistic simulation would spread the ICM particles throughout the cluster so that the galaxy runs all its orbital time through the medium. However, as noted by Abadi et al. (1999), ICM particles that are too massive may become bullets punching holes into the ISM disk. To prevent this, the masses of individual ICM particles should at most be comparable to that of ISM particles. With this assumption and the number of ISM particles set to 12000 , the sphere of $1 \mathrm{Mpc}$ radius of the cluster would need about two million particles of the ICM. Then the calculations would become too time-consuming and complex.

Velocity distribution of the ICM particles is calculated from second moment of the collisionless Boltzmann equation providing isotropy of the model (see e.g. Hernquist 1993):

$\sigma_{R}^{2}=\frac{1}{\rho(R)} \int_{R}^{\infty} \rho(R) \frac{\mathrm{d} \Phi_{\mathrm{tot}}}{\mathrm{d} R} \mathrm{~d} R$,

where $\Phi_{\text {tot }}$ represents the total potential of the cluster's DM and ICM, and $\rho(R)$ is the ICM density. The $\sigma_{R}$ profile is shown in the lower right panel of Fig. 4. Equation (5) is in fact an equation for the hydrostatic equilibrium of the cluster. After a slight initial relaxation, the system reaches a stable state, well before the galaxy enters the ICM distribution. Figure 4 depicts radial profiles of the ICM density (compared to the analytic profile of Eq. (4)), pressure, and speed of sound about 0.5 Gyr before the galaxy arrives. To keep ICM particles within the $140 \mathrm{kpc}$ radius, periodic boundary conditions are introduced such that, when a particle reaches the edge, it continues its course at centrally opposite positions, with preserved velocity vector. Particles of the ICM are treated adiabatically, while particles of the ISM isothermally with $T_{\text {ISM }}=10^{4} \mathrm{~K}$.

Figure 5 shows the initial configuration of the system. Galaxy in face-on orientation relative to the orbit is located at the cluster periphery at $1 \mathrm{Mpc}$ distance from the center, $\boldsymbol{R}=(x, y, z)=[0,0,1] \mathrm{Mpc}$, with no systematic velocity. 

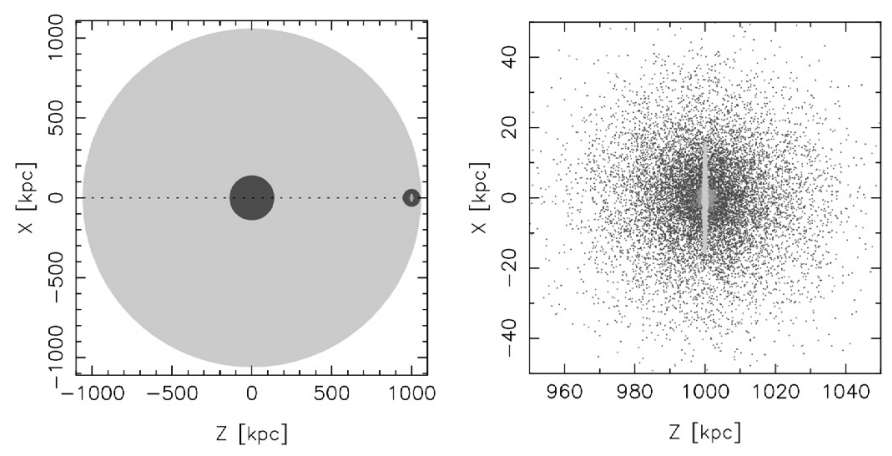

Fig. 5. Initial configuration. Left: galaxy falls face-on from $1 \mathrm{Mpc}$ distance (dashed). The cut-off radius of the ICM is $140 \mathrm{kpc}$ (dark), which outside (light) it operates only gravitationally. Right: zoom on the galaxy with DM halo (dots) and disk+bulge (shaded) components.

Table 2. Parameters of the standard simulation run. Times at which galaxy enters the ICM and reaches the cluster center are stated, together with its velocity in the cluster center.

\begin{tabular}{cccccc}
\hline \hline$N_{\mathrm{ICM}}$ & $\begin{array}{c}R_{\mathrm{c}, \mathrm{ICM}} \\
(\mathrm{kpc})\end{array}$ & $\begin{array}{c}\rho_{0, \mathrm{ICM}} \\
\left(\mathrm{cm}^{-3}\right)\end{array}$ & $\begin{array}{c}T_{R=140 \mathrm{kpc}} \\
\mathrm{Gyr}\end{array}$ & $\begin{array}{c}T_{0} \\
\mathrm{Gyr}\end{array}$ & $\begin{array}{c}v_{0} \\
\left(\mathrm{~km} \mathrm{~s}^{-1}\right)\end{array}$ \\
\hline 120000 & 13.4 & $4 \times 10^{-3}$ & 1.53 & 1.64 & 1260 \\
\hline
\end{tabular}

Consequently, it falls freely towards the cluster center. Thus, in this work, all the orbits are completely radial, free-fall orbits from the distance $R=1 \mathrm{Mpc}$ to the cluster center. For the standard cluster, the galaxy reaches the cluster center after about $1.64 \mathrm{Gyr}$, at a velocity of about $1300 \mathrm{~km} \mathrm{~s}^{-1}$. It means that it moves very supersonically through the ICM in the central regions (cf. Fig. 4), and we expect a strong bow shock to form in front of the galactic disk. Domainko et al. (2006) draw attention to the role of the bow shock: the ram pressure behind the shock is lower since the relative velocities of the ICM particles are smaller there than in front of the shock. On the contrary, Rasmussen et al. (2006) notice that, while the ram pressure is reduced at the shock, $P_{\text {th }}+\rho v^{2}$, where $P_{\text {th }}$ is the thermal ICM pressure behind the shock, stays conserved for an inviscid fluid, and thus the reduction of the ram pressure behind the shock is balanced by an increase in static thermal pressure. Therefore, the force per unit area acting on the disk is close to the pre-shock ram pressure.

Table 2 summarizes the parameters of the standard simulation run. Along the radial orbit, we can compute the ram pressure $p_{\text {ram }}(R)=\rho_{\mathrm{ICM}} v_{\text {gal }}^{2}$ acting on the galaxy as it crosses the ICM. Ram pressure always peaks in the cluster center where the ICM density is highest, and also where the orbital velocity has its maximum. Following Fig. 3, for broader clusters the increase in the ram pressure starts at larger distances from the cluster center. With the same central density $\rho_{0, \mathrm{ICM}}$ the peak value of $p_{\text {ram }}$ is slightly higher for broader clusters due to the higher contribution of the ICM to the overall cluster gravity (Fig. 11, left panel).

In the simulations, we follow the galaxy on its orbit during 2 Gyr. From the very beginning at $1 \mathrm{Mpc}$ distance, the galaxy itself evolves and thus instabilities and spiral arms form. Consequently, the galaxy enters the ICM sphere at about $1.5 \mathrm{Gyr}$ as dynamically evolved.

\section{Simulation method}

We performed 3D tree/SPH $N$-body simulations using GADGET in version 1.1 (Springel et al. 2001). The tree method is a highly effective Lagrangian technique for computing gravity forces in non-homogeneous systems. The particles are arranged into groups hierarchically ordered from the smallest ones containing only one particle up to the biggest one embracing the whole system. The scheme of an oct-tree structure dividing the computational space into a sequence of cubes is used (Barnes \& Hut 1986). Then, the gravitational force exerted by distant groups on a particle is approximated by their lowest multipole moments, which reduces the computational costs to order $O(N \log N)$.

The smoothed particle hydrodynamics (SPH, Lucy 1977; Gingold \& Monaghan 1977) is a Lagrangian method for solving hydrodynamical problems in which real fluids are represented by a set of fluid elements, i.e. particles. The key idea of the SPH is that every particle has an effective radius within which hydrodynamical interactions with neighboring particles operate. Thus the particles are no longer point masses but are smoothed out, and local hydrodynamical properties are interpolated from the local mass distribution. The smoothing sizes of particles are determined to correspond to a radius within which a given number of neighboring particles is contained.

GADGET in version 1.1 resolves only one gaseous phase, and all gas particles occurring in the system are hydrodynamically treated as one group. However, the volume densities of the ISM in the disk and of the surrounding ICM differ by several orders of magnitude, as well as their temperatures. To treat their interaction correctly, the number densities of the ICM and ISM particles, $n_{\mathrm{ICM}}$ and $n_{\mathrm{ISM}}$, should be comparable. Otherwise, the ICM particles located far from the galaxy would be larger in the SPH sense than the ISM particles, since the number of neighboring particles is kept constant throughout the system. When these large ICM particles would later approach the disk, their sizes would be re-evaluated from the local mixing of ICM and ISM particles, and artificial punching effects could follow.

To achieve similar number densities of both gases, a huge number of ICM particles are necessary. With these constraints in mind, we introduce a set of changes into GADGET related to the fact that we need to simulate two different gaseous phases (ICM and ISM) with different spatial resolution. First, the subroutine for searching the neighboring particles is adapted to distinguish between the two phases: the smoothing radii $h$ 's of either ICM or ISM particles are calculated separately from the neighbors of a corresponding phase. In other words, the search continues until $N_{\mathrm{b}}$ proper neighbors are found, while those of the second phase are omitted. Then a reasonable number of ICM particles, that are consequently substantially larger than the ISM, can be used, and their size does not change even when interacting with the disk. It ensures that all the disk area is covered by the ICM particles. Second, the hydrodynamical interaction of the two phases can proceed: both phases are searched together to find all particles with $\left|\boldsymbol{r}_{i j}\right|<\max \left(h_{i}, h_{j}\right)$. A given ISM particle then interacts with all the ICM particles within which smoothing radii it is contained. Third, the adiabatical and isothermal treatments of the ICM and ISM are implemented, respectively.

Figure 6 compares the ISM (black circles) versus ICM (grey circles) particle sizes in the surroundings of the galaxy running through the center of the standard cluster. Face-on and edge-on snapshots are displayed, and the $N_{\mathrm{ICM}}=120000$ and $N_{\text {ISM }}=12000$. Only every tenth particle is plotted, which gives the impression of voids occurring among particles. One notes 

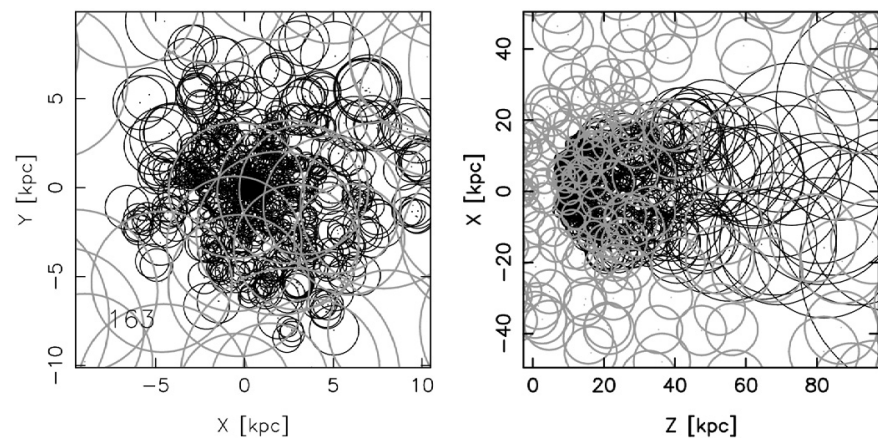

Fig. 6. SPH sizes of the ISM (black) and ICM (grey) particles at the epoch when the galaxy runs through the cluster center. $N_{\mathrm{ICM}}=120000$, $N_{\text {ISM }}=12000$. Left: face-on zoom view of the galaxy surroundings with the ISM particles located within $\pm 2 \mathrm{kpc}$ about the disk plane, and the ICM particles within $\pm 20 \mathrm{kpc}$ about the disk plane. Right: edgeon view of the situation with ICM particles located within $\pm 20 \mathrm{kpc}$ about the disk in the line of sight direction. Only every tenth particle is displayed.

that the size of the ICM particles is kept constant in the disk vicinity.

To achieve a sufficient spatial resolution in the disk, we fix the number of ISM particles $N_{\text {ISM }}$ to an adequate value. In all simulations, the resolution in the disk is then the same. From Fig. 6 it follows that the ICM particles are substantially larger than their ISM counterparts, each of them covering a large part of the disk. That means that a group of close ISM particles lying within the smoothing radius of a given ICM particle feel only the ram pressure of the ICM but no pressure gradients, as would be the case if the sizes of ICM and ISM particles were comparable. In Sect. 7 (Fig. 18), we discuss test runs with varying numbers of ICM particles.

Our simulation approach using only a reasonable number of ICM particles, and thus neglecting the detailed hydrodynamics in the ISM-ICM interaction treatment, has some similarities with the method of Vollmer et al. (2001), who in their stickyparticle simulations include the effect of the ram pressure only analytically as an additional acceleration on the ISM clouds located at the surface of the gas distribution. In our case, of course, the interaction between ICM and ISM is not restricted to the surface layer.

Our approach is more realistic than Vollmer et al. (2001) as far as the ICM is concerned. The ICM phase is treated fully hydrodynamically. Therefore, as discussed later, simulations develop a bow shock that forms in the ICM in front of the galaxy. Consequently, the incoming ICM particles are deflected from their direction to flow around the disk. Moreover, when (from the SPH point of view) small ISM particles are stripped out of the disk, their number density falls and their size increases. In the tail of stripped particles, the sizes of both the ISM and ICM then become comparable, and the hydrodynamical treatment of the tail is correct (see Fig. 6).

\section{Simulation results}

First we describe results of the standard simulation run (see Table 2) of the LM galaxy. This shows how the ISM of the galaxy changes under the influence of a) the ram pressure due to relative motion of the ISM and ICM, b) the gravitational field of the cluster that changes along the orbit, and c) the evolving galactic gravitational field that includes the self-gravity of the ISM disk. We note that no effects of the ram pressure to the stellar disk, bulge or halo galaxy components are observed in the simulations described below.

\subsection{LM galaxy in the standard cluster}

Figure 7 displays snapshots of the ISM disk at five epochs of the galaxy's crossing through the central parts of the cluster. Freely falling from the $1 \mathrm{Mpc}$ distance, it approaches, at time 1.52, the edge of the ICM particle distribution, where ram pressure rises. At $1.64 \mathrm{Gyr}$ it passes through the cluster center and experiences the peak ram pressure. At $1.76 \mathrm{Gyr}$ it leaves the ICM on the other side of its distribution, relaxes after the fadeout of the ram pressure, and further continues towards the second apocenter. Figure 7 shows edge-on and face-on views of the ISM disk, and a wide view of the central parts of the cluster with the ICM particles.

When the galaxy enters the ICM distribution, the local ram pressure affects only the outer disk regions. The ISM from these regions is stripped and thus forms the end of the tail of stripped gas. Later on, more inner parts also get stripped by the increasing ram pressure, filling the central portion of the tail that is clearly visible in Fig. 7. The opening angle of the tail changes with time, which reflects the time evolution of the ram pressure vs. restoring force contest. Schulz \& Struck (2001) describe one effect of the ram pressure enhancing the spiral structure of the disk since particles from inter-arm positions with lower surface densities are more easily removed. This effect is observed at time $1.58 \mathrm{Gyr}$.

The process of stripping is displayed in Fig. 8. It shows the number of ISM particles, i.e. the ISM mass, within different disk radii growing from $1 \mathrm{kpc}$ to $16 \mathrm{kpc}$ with a step of $1 \mathrm{kpc}$, and within a $|z|<1 \mathrm{kpc}$ belt about the disk plane as a function of the orbital time. The uppermost curve shows the ISM mass $M_{\text {bnd }}$ that is bound to the galaxy, i.e. the number of ISM particles with negative total energy. Before the galaxy enters the ICM zone at $\sim 1.53 \mathrm{Gyr}$, no important changes are observed. Once the ram pressure rises, particles from decreasing radii are shifted out of the evaluation belt. With a delay of about 20 Myr after the galaxy's passage through the center, a maximum amount of the ISM is shifted out of the $|z|<1 \mathrm{kpc}$ zone, and curves in Fig. 8 show a minimum corresponding to $49 \%$ of the initial total ISM mass. Particles down to about $4 \mathrm{kpc}$ are influenced. After the minimum, a strong reaccretion of the ISM previously only shifted out of the disk by a short-term effect of the strongest ram pressure, but staying bound to the galaxy occurs. Of course, both the minimum value and the amount of the reaccreted material depend on the definition of the evaluation zone.

With a constant flow of the ICM, Roediger \& Hensler (2005) identify three phases of stripping: (i) an instantaneous phase when the outer disk parts are bent in the wind direction but stay bound to the galaxy; (ii) a dynamic intermediate phase, during which the bending breaks, a part of the ISM is stripped, and another part falls back to the disk; and (iii) a quasi-stable continuous viscous stripping phase when the outer disk layers are peeled off by the Kelvin-Helmholz instability at a rate of $\sim 1 M_{\odot} \mathrm{yr}^{-1}$. The duration of the first phase is $\sim 20-200 \mathrm{Myr}$, while the following second phase is about ten times longer. In our simulations, we observe behavior similar to the first two phases; however, the evolution of the $M_{\mathrm{bnd}}(t)$ and $M_{|z|<1 \mathrm{kpc}}(t)$ under the operation of a peaked ram pressure is different (see Fig. 8). Once the ram pressure rises, the outer disk layers are released, while the inner ones are only shifted from the $|z|<1 \mathrm{kpc}$ zone. Although the rate of the release is lower compared to the 

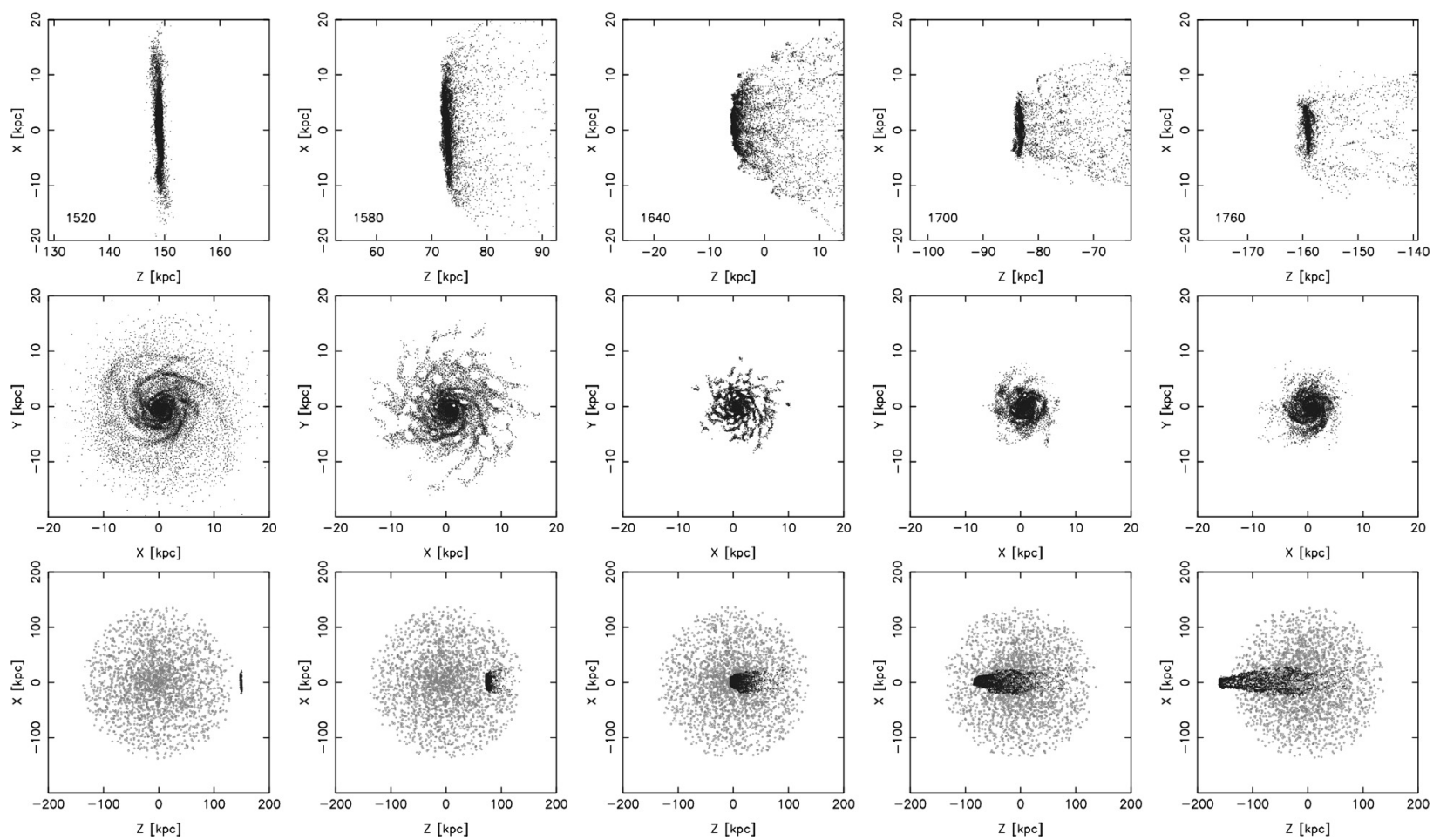

Fig. 7. Time sequence - a galaxy crossing the cluster center. From top down: edge-on projection and face-on cut ( $|z|< \pm 2 \mathrm{kpc})$ of the ISM particles; the tail of the stripped ISM in the central distribution of the ICM particles. The time since the beginning of the free fall of the galaxy from the cluster edge is given in Myr in the first row.

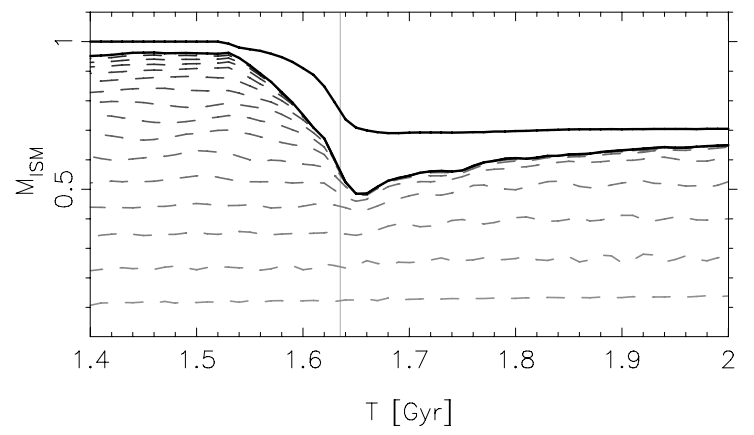

Fig. 8. Standard simulation run. Time evolution of the ISM mass within 16 different disk radii $(1-16 \mathrm{kpc})$ and $|z|<1 \mathrm{kpc}$ (dashed curves). The uppermost curve corresponds to the ISM mass bound to the galaxy. The instant of crossing the cluster center is marked with the vertical line.

shifting, both effects occur simultaneously. When the ram pressure ceases, the shifted, but bound, ISM reaccretes.

From Fig. 8 we determine the final post-stripping mass $M_{\text {final }}$ of the galaxy as the mass of bound ISM at the final simulation time of 2 Gyr: $M_{\text {final }}=0.71 M_{\mathrm{d}, \text { ISM }}$. The stripping radius $r_{\text {strip }}$ is trickier to measure, since all the reaccreting material is not back in the disk at the final time simulated. We estimate it to be $r_{\text {strip }} \sim$ 6 kpc. Initially in Fig. 8 about 5\% of the 12000 ISM particles are, due to relaxation processes and scattering on spiral arms, already outside the region $r<16 \mathrm{kpc},|z|<1 \mathrm{kpc}$ before the ram pressure starts to play a role. But all these particles have negative total energy.

The upper row of Fig. 9 shows the ICM density distribution in the surroundings of the galaxy passing the densest parts of the cluster at times 1.59-1.67 Gyr. The particles are displayed as filled circles with radii equal to their SPH smoothing lengths $h$ 's, where the gray shade corresponds to the density. Due to the supersonic motion, we see a bow shock forming in the ICM. It is most pronounced when the galaxy passes through the very center of the cluster. The bottom row of Fig. 9 shows the ISM density. Due to large differences in the density between the disk and the tail, the grey-scale is logarithmic. Note the growth of ISM particles when they are stripped from the disk. In the Appendix, Fig. A.1 shows the ICM and ISM density distributions for a simulation with $N_{\mathrm{ICM}}=480000$.

Figure 10 shows the velocity vectors of the ICM particles interacting with the ISM disk. At the surface of the disk, ICM decelerates from more than $1000 \mathrm{~km} \mathrm{~s}^{-1}$ to less than $100 \mathrm{~km} \mathrm{~s}^{-1}$. We can also see the deflection of the ICM flow to the sides along the shock, and a low-velocity ICM in the shadow behind the galaxy. In Fig. A.2 of the Appendix the ICM velocity field for the case of $N_{\mathrm{ICM}}=480000$ is shown.

\subsection{LM galaxy in various clusters}

As we have seen in Fig. 8, the stripping of the ISM is a dynamical process depending strongly on the shape of the ram pressure peak. Therefore, we present the results of a set of twenty-five simulations of the LM-type galaxy crossing clusters with varying distributions of the ICM.

\subsubsection{Results from the simulations}

Multiplying the standard values of the parameters $R_{\mathrm{c}, \mathrm{ICM}}$ and $\rho_{0, \text { ICM }}$ by factors of $0.25,0.5,1,2$, and 4 , we study the effects of a varying width and height of the ICM density peak on the stripping results. Figure 11 illustrates the ram pressure profiles corresponding to the ICM distribution with varying widths but a constant height (left), and varying heights but a constant width (right). Figure 12 shows the stripping results of nine runs 

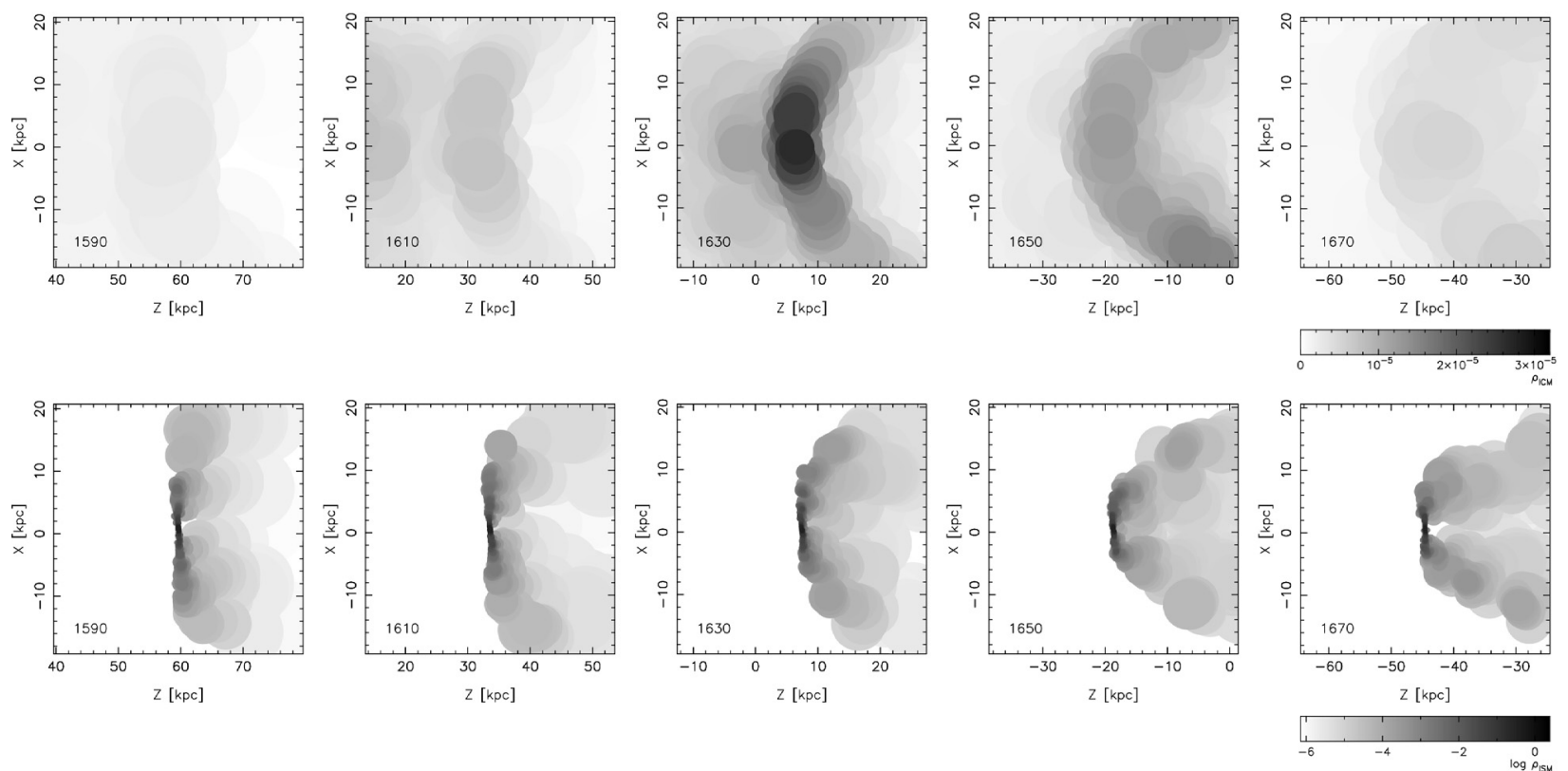

Fig. 9. Density of the ICM in the surroundings of the LM-type galaxy (top) and of the ISM (bottom). A bow shock forms in front of the disk. Individual particles are displayed as filled circles with radii equal to their SPH smoothing sizes $h$ 's. The scale of the ISM density is logarithmic due to large differences in the disk plane and in the tail of stripped particles. The time in Myr is given in the left lower corner of frames.
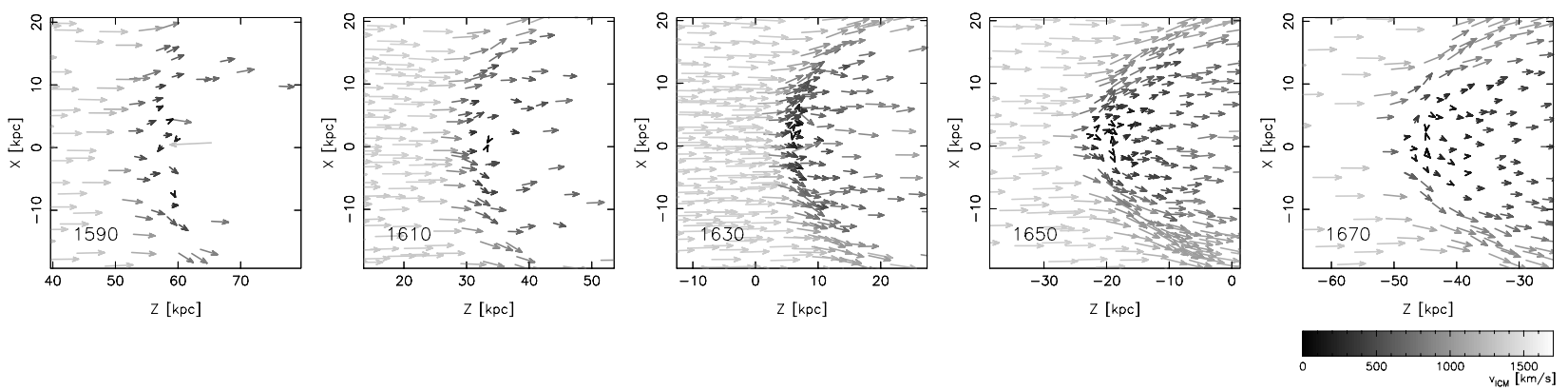

Fig. 10. Flow of the ICM particles around the LM-type galaxy. Vectors of the relative velocity of ICM particles interacting with the ISM disk are displayed.
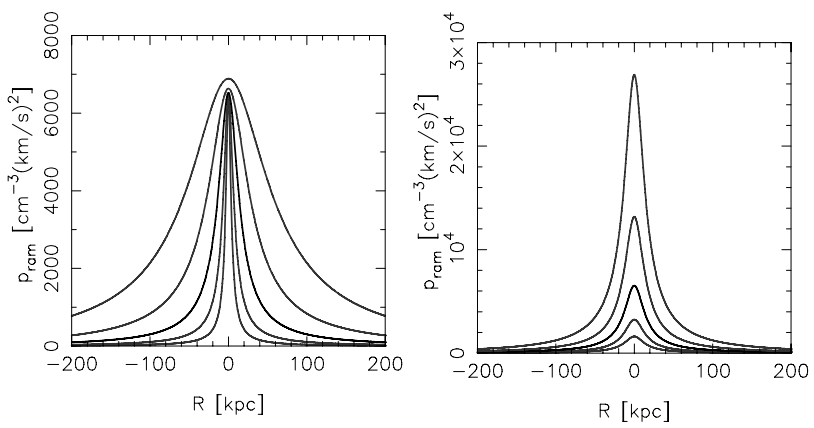

Fig. 11. Central views of the ram pressure peaks with a fixed $\rho_{0, \mathrm{ICM}}=$ $4 \times 10^{-3} \mathrm{~cm}^{-3}$ and $R_{\mathrm{c}, \mathrm{ICM}}=0.25,0.5,1,2,4 \times 13.4 \mathrm{kpc}($ left $)$, and a fixed $R_{\mathrm{c}, \mathrm{ICM}}=13.4 \mathrm{kpc}$ and $\rho_{0, \mathrm{ICM}}=0.25,0.5,1,2,4 \times 4 \times 10^{-3} \mathrm{~cm}^{-3}$ (right).

from the above set of twenty-five simulations. Individual panels are like those in Fig. 8 and correspond to $\rho_{0, \mathrm{ICM}}=0.25,1$, $4 \times 4 \times 10^{-3} \mathrm{~cm}^{-3}$ (from left to right) and $R_{\mathrm{c}, \mathrm{ICM}}=0.25,1$, $4 \times 13.4 \mathrm{kpc}$ (from top to bottom).

The main stripping event is rather short in all the cases and takes 100 to 200 Myr. Going from the upper left corner to lower right of Fig. 12, the stripping expands to more and

more inner parts of the galaxy. It means that both increasing width and height of the ICM density peak support the stripping. Nevertheless, the process of stripping itself is different in either direction:

a) following a row, i.e. for a fixed value of the $R_{\mathrm{c}, \mathrm{ICM}}$, the stripping of the ISM deepens with growing value of the central density. In the upper row, the ram pressure peak is narrow, with $R_{\mathrm{c}, \mathrm{ICM}}=3.4 \mathrm{kpc}$. If the peak is not high (left), most of the ISM remains inside the $|z|<1 \mathrm{kpc}$ layer. About $\sim 12 \%$ of the ISM has been shifted out of the layer, but almost all of it remains gravitationally bound to the galaxy and later will be reaccreted. The wiggles seen on the curves are $z$-oscillations of the reaccreting material around the disk plane. If the ram pressure pulse is high enough (right), it kicks the disk abruptly, which accelerates the outer ISM directly to $v_{\text {esc }}$ and only shifts the inner ISM out of the disk plane. Consequently, $\sim 52 \%$ of the ISM moves out of the $|z|<1 \mathrm{kpc}$ layer, but more than a half of it, i.e. $\sim 30 \%$ of the original amount of the ISM, remains gravitationally bound and is later reaccreted. It may be seen as a deep notch with the deepest part at $T \sim 1.65$ Gyr.

b) Following a column, i.e. for a fixed value of the central density, the stripping begins in wider clusters at earlier times, 

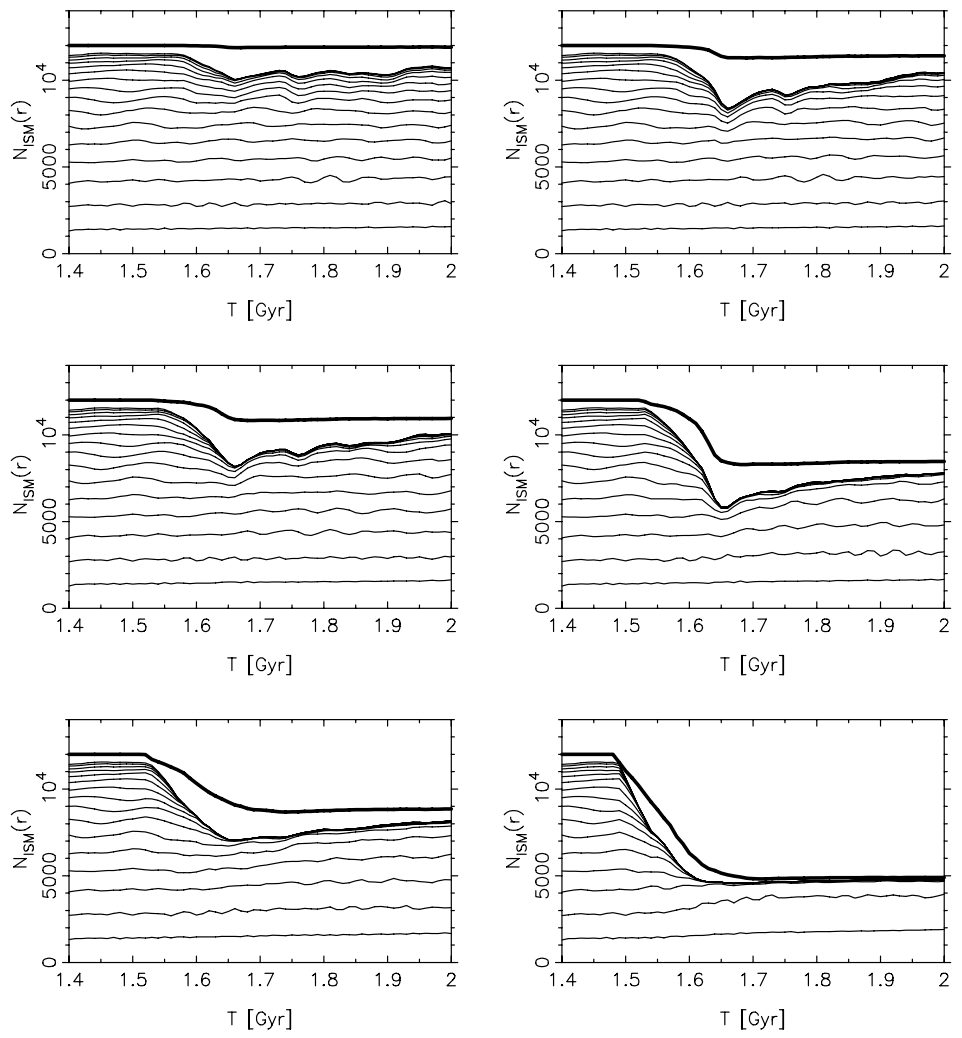
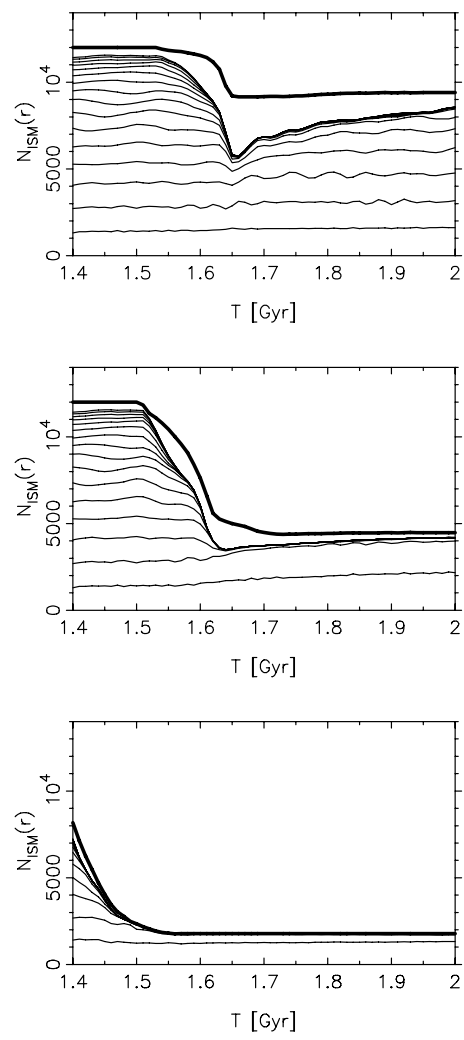

Fig. 12. Stripping of the LM-type galaxy crossing clusters with varying ICM distribution: from left to right: $\rho_{0, \mathrm{ICM}}=0.25,1,4 \times 4 \times 10^{-3} \mathrm{~cm}^{-3}$, from top to bottom: $R_{\mathrm{c}, \mathrm{ICM}}=0.25,1,4 \times 13.4 \mathrm{kpc}$. See Fig. 8 for details. Simulations in the same row thus have a constant value of the $R_{\mathrm{c}, \mathrm{ICM}}$ parameter, and simulations in the same column keep the $\rho_{0, \mathrm{ICM}}$ value. The central panel corresponds to Fig. 8.

since the value of the ram pressure needed to affect the outer ISM shifts to greater distances from the cluster center. Thus, the minimum of the ISM mass in the evaluation zone $|z|<1 \mathrm{kpc}$ broadens. Broad clusters, with $R_{\mathrm{c}, \mathrm{ICM}}=53.6 \mathrm{kpc}$, are not only much more effective in removing the ISM particles outside the $|z|<1 \mathrm{kpc}$ layer, but the more extended ram pressure pulse is also able to accelerate more ISM and remove it from the parent galaxy.

Table 3 quantifies the stripping results of the set of twenty-five simulations and adds values of parameters characterizing individual runs. One sees that in a small (low and narrow) cluster, poor in ICM, where $\rho_{0, \mathrm{ICM}}=1 \times 10^{-3} \mathrm{~cm}^{-3}$ and $R_{\mathrm{c}, \mathrm{ICM}}=3.4 \mathrm{kpc}$, the LM-type galaxy is stripped of at most $1 \%$ of its ISM. It happens between the orbital time 1.53 and 1.64 Gyr. On the other hand, in a large (high and wide) cluster with a lot of ICM, where $\rho_{0, \mathrm{ICM}}=1.6 \times 10^{-2} \mathrm{~cm}^{-3}$ and $R_{\mathrm{c}, \mathrm{ICM}}=53.6 \mathrm{kpc}$, the stripping of $85 \%$ of the ISM is achieved between orbital times 1.37 and $1.56 \mathrm{Gyr}$.

Figure 13 summarizes the results of the set of simulations. The general trends of galaxies crossing wider and higher ICM density peaks losing a growing amount of the ISM, as well as the reaccretion weakening, are clearly visible. In clusters with a constant width, the stripping starts within a short time range, while it occurs earlier in wide clusters, where galaxies fall faster towards the cluster center and the ICM has a greater extent.

\subsubsection{Comparison with the criterion of Gunn \& Gott}

Focusing in Table 3 on values of the column density of the encountered ICM, we note that the passages through clusters with a similar amount of the encountered ICM show a similar amount of the stripped ISM. Figure 14 compares the process of stripping in five clusters with $\Sigma_{\mathrm{ICM}} \sim(5-6) M_{\odot} / \mathrm{pc}^{2}$ which are in Table 3 marked in boldface. In their case, $M_{\text {strip }}=22 \%, 26 \%$, $29 \%, 27 \%$, and $26 \%$. However, the stripping history differs: in a narrow cluster with a high ICM density peak, there is a lot of ISM shifted out of the $|z|<1 \mathrm{kpc}$ zone, but this is reaccreted later. In a broad cluster with a rather shallow density peak, more ISM stays near the galaxy symmetry plane and one sees less of the reaccretion.

The two extreme cases substantially differ in the peak ram pressure; in the first case, it is almost $16 \times$ higher compared to the second case. Gunn \& Gott (1972) criterion (Eq. (1)) would predict a completely different amount of stripping in these cases. However, as we argue later, the final stripping depends on the column density of the encountered ICM, which is similar in the two cases. In Fig. 19 a comparison of the simulation results with Gunn \& Gott (1972) predictions can be seen.

In the left panel of Fig. 15, the dependence of the remaining gas mass fraction $M_{\text {final }}=1-M_{\text {strip }}$ on the extent of the ICM distribution in the cluster is plotted. For increasing $R_{\mathrm{c}, \mathrm{ICM}}$, the degree of stripping becomes less and less dependent on the size of the ICM distribution (characterized by the $R_{\mathrm{c}, \mathrm{ICM}}$ itself) and approaches a constant, which depends on the value of $\rho_{0, \text { ICM }}$. This depends on whether or not the ISM is accelerated during the ram pressure pulse to the escape velocity. The critical size of the active region $\Delta R_{\mathrm{c}}$ is $\Delta R_{\mathrm{c}}=v_{\mathrm{esc}} v \Sigma_{\mathrm{ISM}} / p_{\text {ram }}^{\mathrm{eff}}$, where $p_{\text {ram }}^{\text {eff }}$ is an effective value of the ram pressure. Here we take $p_{\text {ram }}^{\text {eff }}=p_{\text {ram }}^{\text {max }} / 2$. With $\Sigma_{\text {ISM }}=10^{21} \mathrm{~cm}^{-2}, v_{\text {esc }}=400 \mathrm{~km} \mathrm{~s}^{-1}$, $p_{\mathrm{ram}}^{\max }=6000 \mathrm{~cm}^{-3} \mathrm{~km}^{2} \mathrm{~s}^{-2}$, and $v=1300 \mathrm{~km} \mathrm{~s}^{-1}$, we get $\Delta R_{\mathrm{c}} \approx$ $60 \mathrm{kpc}$. This corresponds nicely to $R_{\mathrm{c}, \mathrm{ISM}}=\Delta R_{\mathrm{c}} / 2=30 \mathrm{kpc}$ (see Fig. 15), where $M_{\text {final }}$ loses its dependence on $R_{\mathrm{c}, \text { ISM }}$ and converges to the Gunn \& Gott (1972) prediction given with Eq. (1). 
Table 3. Summary of stripping results of the set of twenty-five simulations with the LM-type galaxy crossing clusters with varying values of the $R_{\text {c.ICM }}$ and $\rho_{0, \text { ICM }}$ parameters. Stripping radius $r_{\text {strip }}$ of the ISM disk, mass of the stripped ISM $M_{\text {strip }}$, minimum mass of the ISM within the evaluation zone, and mass of the reaccreted ISM $M_{\text {accr }}$ are stated. $M_{\text {strip }}+M_{\min }+M_{\text {accr }}=100 \%$. Values of peak ram pressure $p_{\text {ram }}^{\max }$, column density of the encountered ICM $\Sigma_{\mathrm{ICM}}$, and mass of the ICM within central part of clusters $M_{\mathrm{ICM}}$ are added.

\begin{tabular}{|c|c|c|c|c|c|c|c|c|}
\hline $\begin{array}{l}R_{\mathrm{c}, \mathrm{ICM}} \\
(\mathrm{kpc}) \\
\end{array}$ & $\begin{array}{c}\rho_{0, \mathrm{ICM}} \\
\left(10^{-3} \mathrm{~cm}^{-3}\right) \\
\end{array}$ & $\begin{array}{l}M_{\mathrm{ICM}}^{R<140 \mathrm{kpc}} \\
\left(10^{11} M_{\odot}\right)\end{array}$ & $\begin{array}{l}\sum_{\mathrm{ICM}}^{R<140 \mathrm{kpc}} \\
\left(M_{\odot} / \mathrm{pc}^{2}\right)\end{array}$ & $\begin{array}{c}p_{\mathrm{ram}}^{\max } \\
\left(\mathrm{cm}^{-3} \mathrm{~km}^{2} \mathrm{~s}^{-2}\right)\end{array}$ & $\begin{array}{r}r_{\text {strip }} \\
(\mathrm{kpc})\end{array}$ & $\begin{array}{c}M_{\text {strip }} \\
(\%) \\
\end{array}$ & $\begin{array}{c}M_{\min } \\
(\%) \\
\end{array}$ & $\begin{array}{c}M_{\mathrm{accr}} \\
(\%) \\
\end{array}$ \\
\hline \multirow{5}{*}{3.4} & 1 & 0.02 & 0.38 & 1612 & 13.8 & 1 & 84 & 15 \\
\hline & 2 & 0.04 & 0.76 & 3231 & 12.8 & 2 & 78 & 20 \\
\hline & 4 & 0.08 & 1.53 & 6453 & 11.8 & 5 & 70 & 25 \\
\hline & 8 & 0.16 & 3.05 & 12949 & 10.3 & 12 & 59 & 29 \\
\hline & 16 & 0.32 & 6.10 & 25900 & 7.5 & 22 & 48 & 30 \\
\hline \multirow{5}{*}{6.7} & 1 & 0.06 & 0.74 & 1615 & 12.3 & 3 & 75 & 22 \\
\hline & 2 & 0.11 & 1.47 & 3233 & 11.2 & 7 & 68 & 25 \\
\hline & 4 & 0.22 & 2.94 & 6480 & 8.7 & 14 & 57 & 29 \\
\hline & 8 & 0.44 & 5.87 & 13014 & 7.1 & 26 & 47 & 27 \\
\hline & 16 & 0.89 & 11.76 & 26229 & 4.5 & 41 & 36 & 23 \\
\hline \multirow{5}{*}{13.4} & 1 & 0.15 & 1.39 & 1618 & 10.8 & 9 & 68 & 23 \\
\hline & 2 & 0.30 & 2.78 & 3245 & 8.4 & 16 & 59 & 25 \\
\hline & 4 & 0.61 & 5.56 & 6524 & 6.3 & 29 & 49 & 22 \\
\hline & 8 & 1.21 & 11.13 & 13182 & 4.1 & 43 & 40 & 17 \\
\hline & 16 & 2.43 & 22.26 & 26894 & 2.6 & 63 & 29 & 8 \\
\hline \multirow{5}{*}{26.8} & 1 & 0.39 & 2.56 & 1625 & 8.8 & 16 & 64 & 20 \\
\hline & 2 & 0.78 & 5.12 & 3272 & 7.0 & 27 & 55 & 18 \\
\hline & 4 & 1.57 & 10.25 & 6631 & 5.0 & 44 & 44 & 12 \\
\hline & 8 & 3.14 & 20.50 & 13608 & 2.8 & 62 & 31 & 7 \\
\hline & 16 & 6.28 & 40.99 & 28604 & 2.5 & 80 & 20 & 0 \\
\hline \multirow{5}{*}{53.6} & 1 & 0.90 & 4.52 & 1641 & 7.7 & 26 & 59 & 15 \\
\hline & 2 & 1.80 & 9.04 & 3336 & 5.0 & 42 & 47 & 11 \\
\hline & 4 & 3.61 & 18.08 & 6888 & 3.5 & 59 & 38 & 3 \\
\hline & 8 & 7.21 & 36.16 & 14637 & 2.9 & 76 & 24 & 0 \\
\hline & 16 & 14.42 & 72.29 & 32722 & 2.4 & 85 & 15 & 0 \\
\hline
\end{tabular}
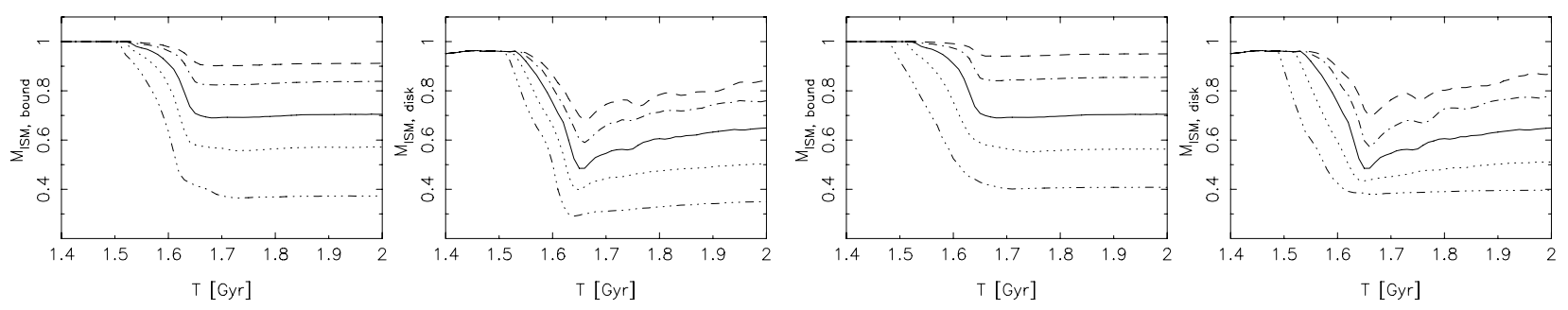

Fig. 13. Stripping of the LM-type galaxy in central parts of clusters with: (left pair) fixed $R_{\mathrm{c}, \mathrm{ICM}}=13.4 \mathrm{kpc}$ and varying $\rho_{0, \mathrm{ICM}}: 0.25,0.5,1,2$, and $4 \times 4 \times 10^{-3} \mathrm{~cm}^{-3}$ (from top to bottom) and (right pair) with fixed $\rho_{0, \mathrm{ICM}}=4 \times 10^{-3} \mathrm{~cm}^{-3}$ and varying $R_{\mathrm{c}, \mathrm{ICM}}: 0.25,0.5,1,2$, and $4 \times 13.4 \mathrm{kpc}($ from top to bottom). Evolution of the ISM mass bound to the galaxy or staying within $r<16 \mathrm{kpc}$ and $|z|<1 \mathrm{kpc}$ about the disk plane is displayed. Reaccretion of the stripped ISM is observable.

Thus our results give much less stripping compared to Gunn \& Gott (1972) in clusters with a narrow ICM peak, while converging to its predictions in extended ICM clusters.

The convergence of the stripping level is apparent from Fig. 15, central panel, which depicts the final ISM mass as a function of the column density of the encountered ICM. Clearly, the higher the $\Sigma_{\text {ICM }}$, the more the galaxy is stripped; however, the dependence is not linear. The decline in the curve corresponds to the increasing duration of the ICM-ISM interaction and to the resulting stripping of more and more strippable elements. The final saturation than equals the Gunn \& Gott prediction when all the elements with $f_{\text {rest }}<p_{\text {ram }}^{\max }$ are stripped. The shape of the dependence is unique for the given orbit, galaxytype, and the cluster potential. Then, knowing from observations the galaxy's velocity and mass, along with the distribution of the cluster DM and $\Sigma_{\text {ICM }}$, one can estimate the stripping and find limits on the Gunn \& Gott criterion. Figure 15, right panel,
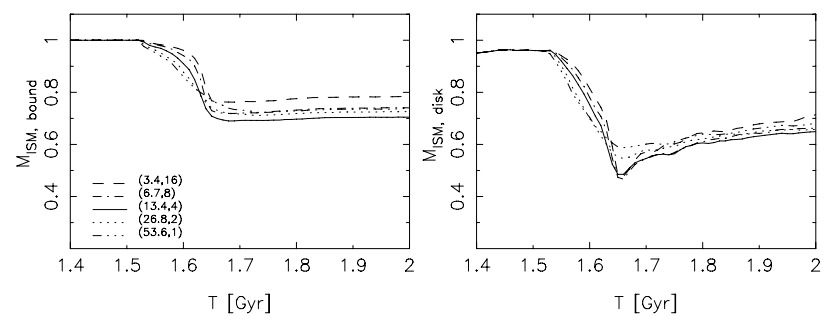

Fig. 14. Stripping of the LM-type galaxy in different clusters but with a similar amount of the encountered ICM, $\Sigma_{\mathrm{ICM}} \sim(5-6) M_{\odot} / \mathrm{pc}^{2}$. Curves correspond to $\left(R_{\mathrm{c}, \mathrm{ICM}}, \rho_{0, \mathrm{ICM}}\right)$ pairs in units $\left(\mathrm{kpc}, 10^{-3} \mathrm{~cm}^{-3}\right)$. The ISM mass bound to the galaxy (left) or staying within $r<16 \mathrm{kpc}$ and $|z|<1 \mathrm{kpc}$ layer about the disk plane (right) are displayed.

further shows the reciprocal of the ISM final mass as a function of $\Sigma_{\mathrm{ICM}}$ for LM-type galaxy. We try to fit the dependence 

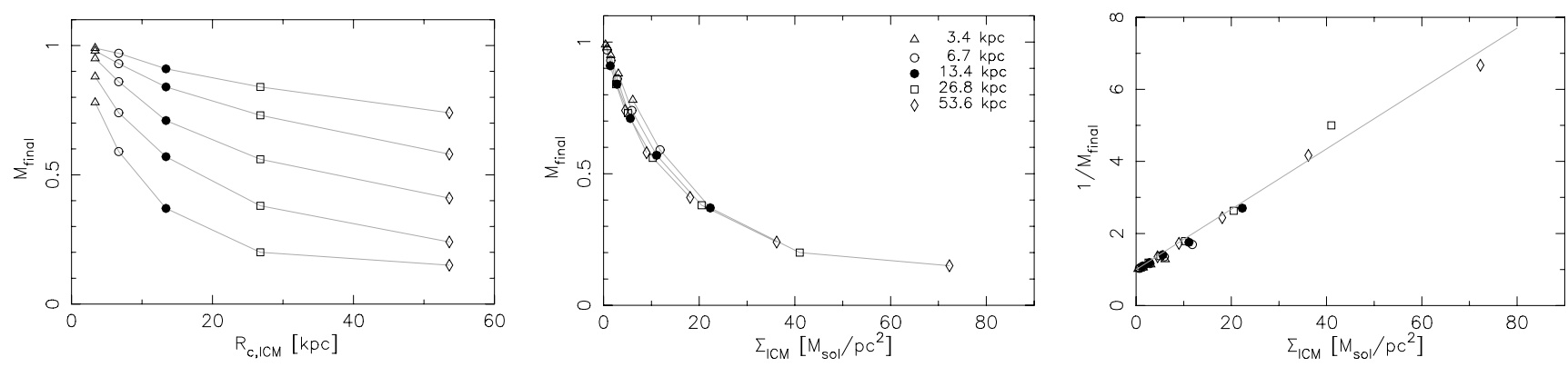

Fig. 15. Left: final ISM mass fraction $M_{\text {final }}$ as a function of $R_{\text {c,ICM }}$ for the LM-type galaxy. The lines correspond to different values of the $\rho_{0, \text { ICM }}: 1$, $2,4,8$, and $16 \times 10^{-3} \mathrm{~cm}^{-3}$ (from top down); see legend in the central panel. Center: $M_{\text {final }}$ as a function of the column density of the encountered ICM, $\Sigma_{\mathrm{ICM}}$, for the LM-type galaxy. Right: inverse value of $M_{\text {final }}$ as a function of $\Sigma_{\mathrm{ICM}}$.
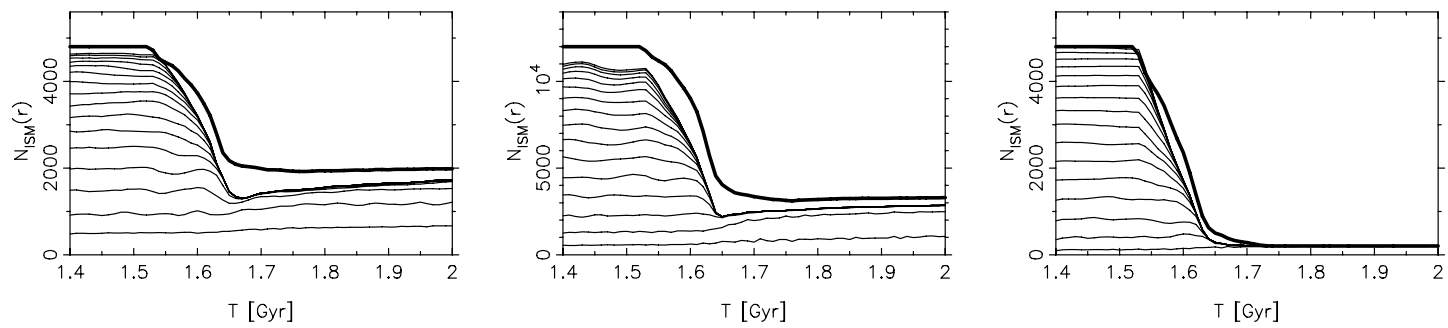

Fig. 16. Evolution of the stripping of the Lm, EM, and Em galaxy types (from left to right) during their crossing of the standard cluster.

with $M_{\text {final }}^{-1}=k \Sigma_{\mathrm{ICM}}+1$, where $k \sim 0.09$. The quantity $M_{\text {final }}^{-1}$ in fact corresponds to $M_{\mathrm{ISM} \text {,init }} / M_{\mathrm{ISM} \text {,final }}=M_{\mathrm{HI} \text {,expected }} / M_{\mathrm{HI}, \mathrm{observed}}$, whose logarithm then gives the deficiency DEF introduced by Solanes et al. (1996).

\subsection{Various galaxy types}

A comparison of the models Lm, EM, and Em in the standard cluster is shown in Fig. 16. The LM-type galaxy has a stripping radius of about $6 \mathrm{kpc}$ and loses about $29 \%$ of its original ISM. For Lm and EM-type galaxies the stripping radii move closer to the galaxy center, to about $3.9 \mathrm{kpc}$ and $2.9 \mathrm{kpc}$, respectively. The Em-type galaxy is stripped almost completely. As summarized in Table 4, the LM, Lm, EM, and Em galaxy types lose about $29 \%, 59 \%, 69 \%$, and $96 \%$ of its original ISM during the face-on motion through the cluster with standard ICM distribution.

We may ask why the amount of the stripping is smaller in the case of the Lm-type compared to the EM-type, when the maximum restoring force is stronger for the EM galaxy except the innermost radii (see Fig. 2, left panel). However, in the range $(1-4) \mathrm{kpc}$, where the stripping radii of the two cases reside, the maxima of the restoring forces are almost equal. Further, the surface density of the ISM is lower, and the escape velocity is higher in the EM-type compared to the Lm-type. But, as we see later, the combination of a lower escape velocity and a higher $\Sigma_{\text {ISM }}$ in the Lm-type does not explain less stripping compared to the EM-type.

As shown in the middle panel of Fig. 2, the maxima of the restoring force over $z$-direction occur substantially closer to the disk plane in the case of the Lm galaxy, especially at small galactic radii. Thus, the rise in the restoring force behind the disk is much steeper in the Lm galaxy than in the EM model. In both cases, the galaxies move through the narrow standard cluster. Consequently, the ISM of the Lm-type galaxy is only shifted slightly out of the disk by the short ICM wind pulse. On the contrary, the ISM in the EM-type galaxy is more easily shifted to
Table 4. Stripping results for different galaxy types in the standard cluster.

\begin{tabular}{ccccc}
\hline \hline Gal. type & $\begin{array}{c}R_{\text {strip }} \\
(\mathrm{kpc})\end{array}$ & $\begin{array}{c}M_{\text {strip }} \\
(\%)\end{array}$ & $\begin{array}{c}M_{\min } \\
(\%)\end{array}$ & $\begin{array}{c}M_{\text {accr }} \\
(\%)\end{array}$ \\
\hline LM & 6.3 & 29 & 49 & 22 \\
Lm & 3.9 & 59 & 27 & 14 \\
EM & 2.9 & 73 & 18 & 9 \\
Em & 0.2 & 96 & 4 & 0 \\
\hline
\end{tabular}

higher $z$ 's by the same ram pressure pulse, and is thus accelerated towards the $v_{\text {esc }}$ more effectively. This difference raises an interesting physical possibility: if the peak in the restoring force is a long way from the midplane, the ram pressure is able to do work on disk gas, building up its kinetic energy, before the gas encounters the peak of the restoring force. The stored kinetic energy could then be sufficient to get (or helping to do so) the gas out of the galaxy, even if the ram pressure alone is insufficient to do this.

The difference in the amount of ICM momentum delivered to the ISM also has hydrodynamical causes: the higher density of the ISM leads to creation of a larger and stronger bow shock, better protecting the ISM against the incoming ICM. That is more effectively deflected to the sides, and a fraction of the ICM pressure is directed to the sides, thereby decreasing the postshock pressure. Then the ISM in Lm-type gets less ICM momenta compared to EM galaxy.

Figure 17 displays five snapshots of the Lm and EM galaxies during their passage through the central parts of the standard cluster. The ISM disk of the EM-type galaxy is much thicker than that of the Lm-type due to its higher bulge-to-disk mass ratio. This leads to another possible explanation of the more effective stripping of the EM galaxy, where its thicker disk is more vulnerable to the ram pressure. 

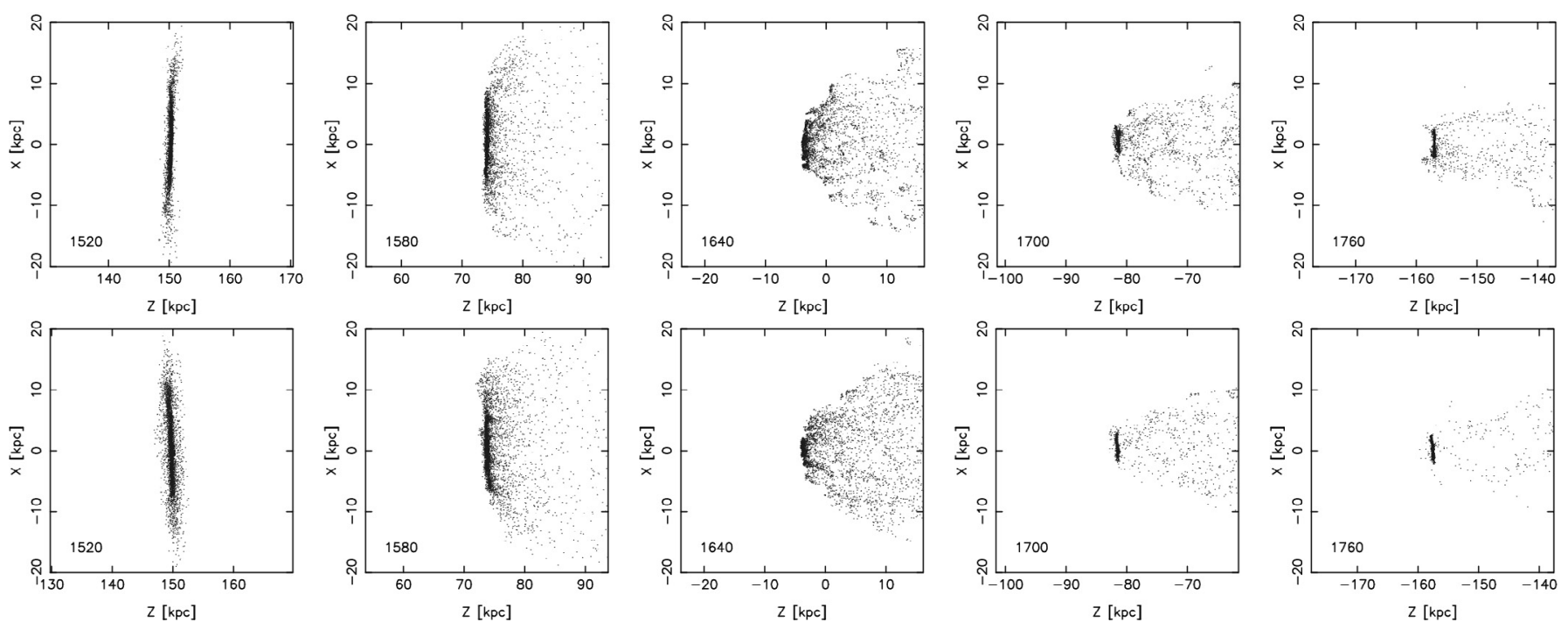

Fig. 17. Edge-on snapshots of the stripping of the Lm (top) and EM (bottom) galaxies. The ISM disk of the EM galaxy type is clearly thicker in comparison with the Lm-type before they enter the ICM distribution.

\section{Simulations with varying SPH sizes of ICM particles}

To test the influence of the number of ICM particles, we vary $N_{\text {ICM }}$ by a multiplicative factor $\alpha$. Then, since the SPH number of neighbors is fixed, the smoothing lengths evolve as $h^{\prime}=$ $h \alpha^{-1 / 3}$. As the number of ICM particles in the simulation increases, the hydrodynamical interactions are treated in a better way, since the mutual sizes of ICM and ISM particles approach. We have performed a set of test runs with the LM-type galaxy crossing the standard cluster with a number of ICM particles varying as $480000,120000,24000$, and 12000 . Figure 18 displays the results - the time evolution of the ISM mass bound to the galaxy (top), and the ISM mass located within the $r<16 \mathrm{kpc}$, $|z|<1 \mathrm{kpc}$ zone (bottom). Although the number of ICM particles differs by a factor 40 in the extreme cases, which corresponds to about a 3.4-fold difference in the SPH particle sizes, only a small difference in the final stripping results is observed in Fig. 18.

Since all particles meeting the condition $\left|\boldsymbol{r}_{i j}\right|<\max \left(h_{i}, h_{j}\right)$ are used to evaluate the pressure gradient at the disk, the ISM particles still feel the static pressure of the ICM behind the bow shock, even though the SPH sizes of the ICM particles may be much greater than those of the ISM. This view is supported by Fig. 18, since changing the number of ICM particles does not alter the stripping rate. The dominant process governing the sweeping in the case of supersonic ISM-ICM interaction is the ram pressure.

Figure 18 also shows that, due to smoothing of the ICM peak by increasing sizes of the particles, shifting of the ISM out of the $|z|<1 \mathrm{kpc}$ evaluation zone is steeper. Apart from changing sizes of the ICM particles, the growing value of the $N_{\text {ICM }}$ causes different mass ratios of individual ICM vs. ISM particles: 10.3, 5.2, 1 , and 0.26 .

\section{Analytical model}

After presenting our numerical results, we now consider the interaction of the galactic ISM with the ICM from an analytic point of view.

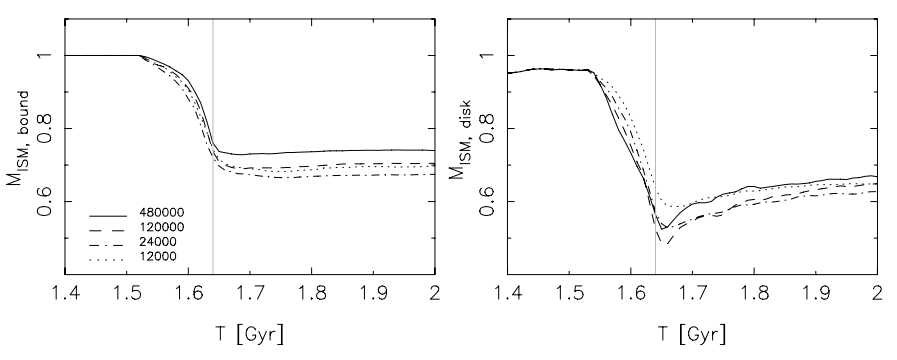

Fig. 18. Simulation results of the LM-type galaxy crossing the standard cluster with a varying number of ICM particles. Total mass of the bound ISM (left) and of the ISM located within $r<16 \mathrm{kpc},|z|<1 \mathrm{kpc}$ zone about the disk plane (right) are displayed as functions of the orbital time. The vertical line indicates the instant of the passage through the cluster center.

\subsection{The equation of motion of an ISM element}

We describe the trajectory of an individual ISM element originally sitting in the disk plane, as it moves under the influence of the cluster gravity forces, the restoring force of the galaxy, and the ICM pressure force.

As shown in the simulations, a bow shock forms when the incoming ICM arrives at the galaxy's disk. Consequently, the ICM decelerates at the shock, transferring part of its momentum to the galaxy, and flows along the shock envelope around the disk. The disk itself feels the pressure of the bow shock and thus the ISM is pushed out of the disk plane, against the galaxy's restoring force.

Disregarding the above hydrodynamical effects and considering the face-on case, the equation of motion (EOM) for the movement of an ISM element in the $z$-direction, i.e. vertical to the galactic plane, is

$$
\frac{\mathrm{d}\left(v_{\mathrm{out}} \Sigma_{\mathrm{ISM}}\right)}{\mathrm{d} t}=\rho_{\mathrm{ICM}}\left(v-v_{\text {out }}\right)^{2}-\frac{\partial \Phi}{\partial z} \Sigma_{\mathrm{ISM}},
$$

where $v$ is the galaxy's orbital velocity. The rightmost term is the gravitational restoring force at the current $z$-position of the ISM element, whose current velocity is $v_{\text {out }}$ with respect to the galaxy's rest frame. The ISM element feels a reduced ram pressure because of the relative velocity of the galaxy, and the ICM, 


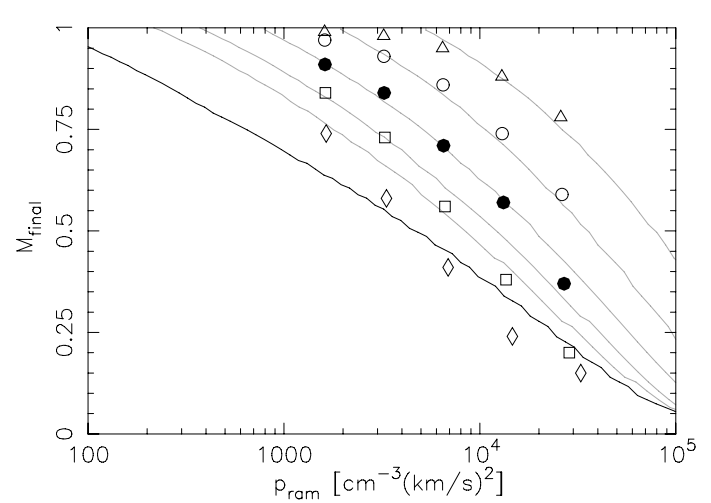

Fig. 19. Final mass fraction $M_{\text {final }}$ of the gas that remains in the disk after the stripping events as a function of the maximum value of the ram pressure $p_{\text {ram }}$. The symbols (see legend in Fig. 15) denote the results of the SPH calculations for five groups of clusters with increasing central density (from left to right), while the gray curves are the corresponding predictions obtained by integration of Eq. (6). The thick solid line refers to the criterion of Gunn \& Gott (1972), Eq. (1).

$v$, is reduced by $v_{\text {out }}$. When $v_{\text {out }}$ exceeds the local value of the escape velocity $v_{\mathrm{esc}}(r, z)$, the element is stripped.

The solution of this equation, together with $\mathrm{d} z / \mathrm{d} t=v_{\text {out }}$, gives the speed and the trajectory of the ISM element in the gravitational field of the galaxy and under the influence of the changing ram pressure. From Eq. (6) one notes that, apart from the actual value of the ram pressure reduced by the local restoring force, the history of the encountered ICM also matters. In the disk of a galaxy, one applies this EOM to the ISM at each galactocentric radius; and by numerical integration, one can follow the fate of each annulus of the disk until it is stripped. The stripping radius $r_{\text {strip }}$ is a radius above which $v_{\text {out }}(r)$ exceeds the value of the escape velocity in the galactic plane. The final mass is then determined as the mass of the ISM enclosed within the stripping radius, according to the density profile in the disk (Eq. (3)).

Figure 19 compares these predictions of Eq. (6) with the results of the SPH simulations, depicting $M_{\text {final }}$ as a function of the peak value $p_{\text {ram }}$ of the ram pressure. One notes that the curves from the simple EOM model match the results obtained by the SPH simulations rather well, in particularly for narrow clusters with $R_{\mathrm{c}, \mathrm{ICM}}=3.4,6.7$, and $13.4 \mathrm{kpc}$. For large clusters, the simple model underestimates the stripping severely. This may be because in Eq. (6) we neglect the deformation of the stripped ISM disk, which would affect the restoring force, or none of the hydrodynamical effects is taken into account. Nonetheless, this very simple approach reproduces the dependence on both the extend and the density of the cluster quite well.

Figure 19 also shows that the criterion of Gunn \& Gott (1972) constitutes the limit for maximum possible stripping. The final mass fractions predicted from the simple approach converge towards the estimates based on Eq. (1) at high values of $R_{\mathrm{c}, \mathrm{ICM}}$ and $\rho_{0, \mathrm{ICM}}$, but they always remain higher. This is because Gunn \& Gott (1972) assume a constant operation of the peak value of the ram pressure and compare it with the maximum restoring force. Therefore, at all radii of the disk, where the (peak) ram pressure exceeds the maximum restoring force, all the ISM elements can be accelerated to the escape velocities. However, in the integration of the EOM, an ISM element may cross the $z$-distance of the maximum restoring force, but the peak of the ram pressure may be over before the element reaches escape velocity.

\subsection{The impulse approximation}

Since our principal intention is not to follow the exact trajectory of the stripped elements but only to identify them and thus obtain the stripping radius (or the final mass), let us introduce an approximative solution of the EOM.

If we consider only those regions where the gas is clearly removed from the galaxy disk, we can obtain a first-order solution by considering the balance of the momentum. Neglecting the restoring force in these outer regions, the momentum per unit surface area attained by the stripped gas of surface density $\Sigma_{\text {ISM }}$ and velocity $v_{\text {after }}$ is

$P_{\mathrm{after}}=v_{\mathrm{after}} \Sigma_{\mathrm{ISM}}=\int \rho_{\mathrm{ICM}} v^{2} \mathrm{~d} t$.

This quantity $P_{\text {after }}$ can be considered as the integrated history of the ram pressure:

$P_{\mathrm{after}}=\Sigma_{\mathrm{ICM}} \frac{\int \rho_{\mathrm{ICM}} v^{2} \mathrm{~d} t}{\int \rho_{\mathrm{ICM}} v \mathrm{~d} t}=\Sigma_{\mathrm{ICM}}\langle v\rangle_{\rho_{\mathrm{ICM}}}$,

where $\Sigma_{\mathrm{ICM}}=\int \rho_{\mathrm{ICM}} v \mathrm{~d} t$ is the column density of the encountered ICM, and $\langle v\rangle_{\rho_{\mathrm{ICM}}}$ is the averaged velocity $v$ along the galaxy's orbit weighted with the local volume density $\rho_{\text {ICM }}$. Then Eq. (7) becomes

$v_{\mathrm{after}}=\langle v\rangle_{\rho_{\mathrm{ICM}}} \frac{\Sigma_{\mathrm{ICM}}}{\Sigma_{\mathrm{ISM}}}$

Of course, the restoring force $f_{\text {rest }}$ can only be neglected in the stripped outer parts of the galaxy. A better approximation of the EOM is to consider the momentum transferred from the inflowing ICM to the ISM element after the element passed the position $z$ with maximum value of the restoring force. Thus, one regards the acceleration of the ISM only when the ram pressure exceeds this maximum. Then, at a given radius,

$v_{\text {after }}=\frac{\int\left(\rho_{\mathrm{ICM}} v^{2}-\left.\frac{\partial \Phi}{\partial z}\right|_{\max } \Sigma_{\mathrm{ISM}}\right) \mathrm{d} t}{\Sigma_{\mathrm{ISM}}}$,

where the integration is taken over a time interval $\left(t_{1}, t_{2}\right)$ when $p_{\text {ram }}>f_{\text {rest,max }}$, i.e. as long as the Gunn \& Gott (1972) criterion (Eq. (1)) is fulfilled. Since the actual value of the restoring force at the position $(r, z)$ is less compared to its maximum value, this obviously overestimates the binding of an element to the galaxy plane.

Figure 20 shows a comparison of the results of the SPH simulations and from the approximative solutions by Eqs. (9) and (10), which are expected to bracket the actual equation. As before, the stripping radius $r_{\text {strip }}$ is the radius above which $v_{\text {after }}(r)$ exceeds the value of the escape velocity in the $z=0$ plane. Then the final mass is determined as the mass of the ISM enclosed within the stripping radius, according to the disk's density profile (Eq. (3)). One notes that the curves from Eq. (9), which neglects the restoring force, indeed overestimate the stripping, while curves based on Eq. (10), which consider only the momentum gained as long as the ram pressure exceeds the maximum restoring force, underestimate the stripping. In any case, the departures from SPH results are larger than with the predictions of the EOM (Eq. (6)), displayed in Fig. 19.

Of course, comparing the full numerical treatment with the predictions of the analytical approach may have several caveats. The most important is that all the hydrodynamical processes, such as the effect of the bow-shock and size of the galaxy 

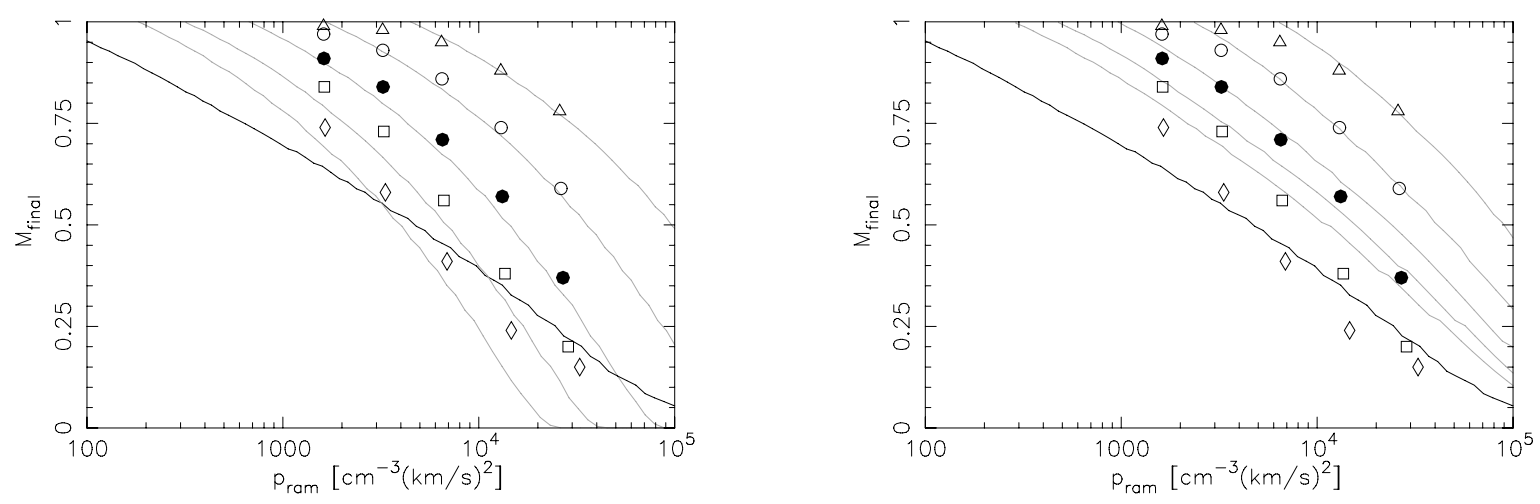

Fig. 20. Numerical results versus analytic predictions of Eqs. (9) (left) and 10 (right) of final remaining gas mass fraction $M_{\text {final }}$ after the stripping events as functions of the maximum value of the ram pressure $p_{\text {ram }}$. The symbols for individual simulations are the same as in Fig. 19. The thick solid line corresponds to Gunn \& Gott (1972), Eq. (1).

shadow, are disregarded in the solution that describes the dynamics of a single ISM element. Furthermore, in the analytic calculations, the density profile of the ISM is assumed to be constant, whereas in the full simulations, processes of relaxation and formation of spiral arms, as well as the progressive stripping, can change the surface density profile of the gas disk. Such changes consequently affect the restoring force and the escape velocity profiles.

Nonetheless, it is interesting that the simple conservation of momentum (Eq. (9)), which on one hand neglects the actual effect of the restoring force and the element's shifting out of the disk and on the other overestimates the escape velocity and changes of the ISM disk due to stripping, can give a good order of magnitude estimate of the stripping, when the variation in the ICM density along the galaxy's path is to be taken into account. This corresponds to the above fit of simulation results with $M_{\text {final }} \sim\left(k \Sigma_{\text {ICM }}+1\right)^{-1}$ : for higher $\Sigma_{\text {ICM }}, v_{\text {after }}$ grows, turning more and more elements to higher than escape velocity $v_{\text {esc }}$. Therefore $M_{\text {final }}$ decreases with growing $\Sigma_{\text {ICM }}$.

The Gunn \& Gott (1972) formula, computed from the maximum ICM density, overestimates the stripping and does not allow for the effect of the finite size of the cluster, i.e. the finite time of interaction. However, in cases with high values of $R_{\mathrm{c}, \mathrm{ICM}}$ and $\rho_{0, \mathrm{ICM}}$, the SPH simulations show an even larger stripping than the Gunn \& Gott (1972) estimate. This may be a consequence of uneven gas distribution in the galaxy disk where spiral-arm inhomogeneities contribute to the ISM removal, as also shown by Quilis et al. (2000).

\section{Comparison to other simulations}

\subsection{Abadi et al. (1999)}

Performing 3D SPH/ $N$-body simulations, Abadi et al. (1999) studied the stripping of spiral galaxies by operating a constant flow of ICM particles in a simulation box of size $60 \mathrm{kpc} \times$ $60 \mathrm{kpc} \times 10 \mathrm{kpc}$ homogeneously filled up with the ICM particles flowing with periodic boundary conditions. Their model is a spiral galaxy exposed to a wind of $1000 \mathrm{~km} \mathrm{~s}^{-1}, 2000 \mathrm{~km} \mathrm{~s}^{-1}$, or $3000 \mathrm{~km} \mathrm{~s}^{-1}$ and of density: $\rho_{\mathrm{c}}=3.37 \times 10^{-3} \mathrm{~cm}^{-3}$, and $\rho_{v}=0.1 \rho_{\mathrm{c}}$. The ram pressure then ranges from about 300 to $30000 \mathrm{~cm}^{-3} \mathrm{~km}^{2} \mathrm{~s}^{-2}$. For the higher value of the ICM density, they estimate the stripping radius of a spiral galaxy to $4 \mathrm{kpc}$, i.e. to about $80 \%$ loss of the galaxy's diffuse gas mass.

\subsection{Quilis et al. (2000)}

In their simulations employing a high-resolution 3D Eulerian code with a fixed grid based on high-resolution shock-capturing method, Quilis et al. (2000) follow the interaction between the hot ICM and the cold ISM. The stellar and DM components are evolved using a particle-mesh code. They model a luminous spiral galaxy similar to the Milky Way or Andromeda. The ICM is modeled as a uniform medium with temperature $T_{\mathrm{ICM}}=10^{8} \mathrm{~K}$ and constant densities $\rho_{\mathrm{c}}=2.6 \times 10^{-3} \mathrm{~cm}^{-3}$ and $\rho_{v}=0.1 \rho_{\mathrm{c}}$. The galaxy moves through the ICM wind at velocities of $1000 \mathrm{~km} \mathrm{~s}^{-1}$ or $2000 \mathrm{~km} \mathrm{~s}^{-1}$. For the Coma-like ICM density and velocity, they estimate the stripping radius as $\sim 3 \mathrm{kpc}$.

\subsection{Vollmer et al. (2001)}

Investigating the role of the ram pressure stripping in the Virgo cluster, Vollmer et al. (2001) employ a method of sticky particles for modeling the warm neutral clouds of the ISM. The effect of the ram pressure is included only analytically as an additional acceleration on the clouds located at the windward side of the gas distribution. Contrary to other simulations discussed in this section, they introduce temporal ram pressure profiles in their simulations instead of a constant ICM wind. They use a spiral galaxy model in a wind creating the ram pressure reaching maximum values ranging from 1000 to $10000 \mathrm{~cm}^{-3} \mathrm{~km}^{2} \mathrm{~s}^{-2}$. Galaxies are considered on slightly elliptical orbits not reaching the very center of the cluster. Due to a high slope of the $\beta$-profile, the ICM density falls steeply with distance $R$ from the cluster center. At the smallest pericenter used $(R \sim 70 \mathrm{kpc})$, the ICM density exceeds about $3 \times 10^{-3} \mathrm{~cm}^{-3}$. In our present simulations, galaxies on completely radial orbits are studied. To achieve similar effects of the ram pressure as Vollmer et al. (2001), we have set $\rho_{0, \text { ICM }}=0.1 \times \rho_{0, \text { ICM }}^{\text {Vollmer }}$ as our standard value (see Sect. 3 ).

Vollmer et al. (2001) show that the ram pressure can lead to a temporary increase in the central gas surface density and that in some cases a strong reaccretion of the atomic gas occurs after the stripping event. They find that the scenario of ram pressure stripping being responsible for the observed H I deficiency is consistent with all H I $21 \mathrm{~cm}$ observations in the Virgo cluster.

The difference between a slightly elliptical orbit with the pericenter distance $R_{\mathrm{p}}$ and a completely radial orbit with the same maximum value of the ICM density encountered, i.e. $\rho_{0, \mathrm{ICM}}^{\mathrm{rad}}=\rho_{\mathrm{ICM}}^{\text {ellipt }}\left(R_{\mathrm{p}}\right)$, and the same $R_{\mathrm{c}, \mathrm{ICM}}$, is in the effective size of the interacting region. The width of the ram pressure peak is larger in the former case than in the latter one. From that it 
Table 5. The list of simulations.

\begin{tabular}{cccc}
\hline \hline Author & Method & ICM flow & galaxy \\
\hline Abadi et al. & SPH & constant & spiral \\
Quilis et al. & 3D finite diff. & constant & spiral \\
Vollmer et al. & sticky particle & changing & spiral \\
Schulz et al. & SPH-AP ${ }^{3} \mathrm{M}$ & constant & dwarf \\
Roediger et al. & 2D finite diff. & constant & spiral \\
Jáchym et al. & SPH & changing & spiral \\
\hline
\end{tabular}

follows that in our simulations the ISM-ICM interactions take place for a shorter time as compared to Vollmer et al. (2001).

\subsection{Schulz \& Struck (2001)}

A model dwarf galaxy in 3D HYDRA (SPH-AP $\left.{ }^{3} \mathrm{M}\right)$ simulations of Schulz \& Struck (2001) is placed in a cubical grid with $100 \mathrm{kpc}$ edge. The galaxy is surrounded with 80000 uniformly distributed ICM particles. The temperature of the ICM is $T_{\mathrm{ICM}}=4.6 \times 10^{5} \mathrm{~K}$, and the density of the wind is $\sim 7.3 \times$ $10^{-5} \mathrm{~cm}^{-3}$. In their face-on models, the galaxy moves with a velocity of $1000 \mathrm{~km} \mathrm{~s}^{-1}$ or $2000 \mathrm{~km} \mathrm{~s}^{-1}$. Then, the stripping radii measured from their Figs. 2 and 14 are $\sim 7.2 \mathrm{kpc}$ and $\sim 5.2 \mathrm{kpc}$.

\subsection{Roediger \& Hensler (2005)}

With a spiral galaxy models and a wide range of ICM conditions, Roediger \& Hensler (2005) perform a parameter study of the ram pressure stripping using 2D Eulerian simulations. Except for the gaseous disk, all components are treated analytically, providing only the gravitational potential. The ICM densities range from $10^{-5}$ to $10^{-3} \mathrm{~cm}^{-3}$, and the resulting ram pressure covers a range from 10 to $10000 \mathrm{~cm}^{-3} \mathrm{~km}^{2} \mathrm{~s}^{-2}$.

\subsection{Summary}

The different simulations are listed in Table 5 and a comparison is shown in Fig. 21. All the models shown, except Schulz \& Struck (2001), use comparable spiral galaxy models. Abadi et al. (1999), Quilis et al. (2000) and Roediger \& Hensler (2005) show high stripping efficiency close to the Gunn \& Gott (1972) prediction, which is due to a constant ICM density adopted. There is no difference in the stripping radius and $M_{\text {final }}$ from $2 \mathrm{D}$ and 3D hydrodynamical simulations and SPH. It seems that the global stripping effect on the galaxy does not depend on the details of the hydrodynamical approach.

The results of Schulz \& Struck (2001) show the same stripping efficiency at lower ram pressures (see Fig. 21), which is, in accordance to Gunn \& Gott (1972), due to lower disk restoring force in the case of dwarf galaxies.

Our models with low values of $R_{\mathrm{c}, \mathrm{ICM}}$ show less stripping. This is mainly due to the effect of changing strength and finite duration of the ram pressure peak. For high values of $R_{\mathrm{c}, \mathrm{ICM}}$ in large clusters with long interaction times, our models approach the Gunn \& Gott (1972) prediction and the results of other models. It demonstrates that our SPH approach gives similar results to SPH simulations of others and to 2D and 3D Eulerian codes.

Simulations by Vollmer et al. (2001) use the changing ram pressure force acting on sticky particles to show results close to Gunn \& Gott (1972) prediction. However, with the similar dependence of the ram pressure on time, we get less stripping. Simulations with sticky particles probably overestimate the stripping efficiency, since this kind of simulation disregards

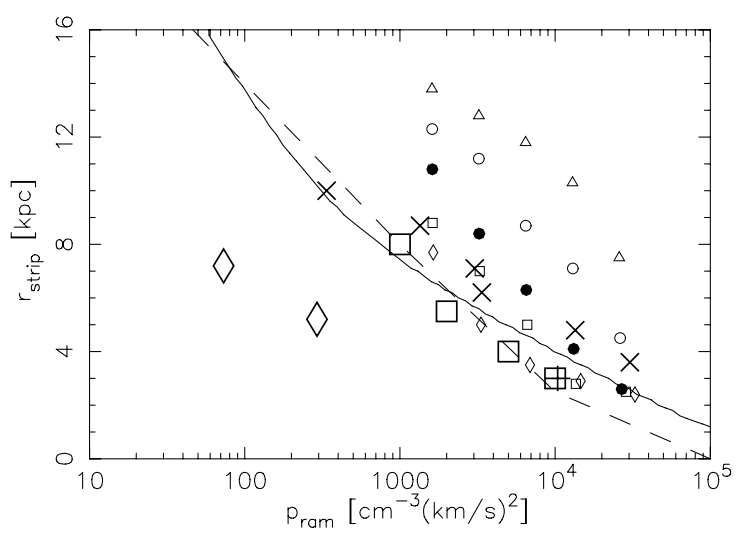

Fig. 21. Stripping radius $r_{\text {strip }}$ versus the peak ram pressure $p_{\text {ram }}^{\max }$ : a comparison with simulations by Abadi et al. (1999) - large $\times$; Quilis et al. (2000) - large plus; Vollmer et al. (2001) - large squares; Schulz \& Struck (2001) - large diamonds; models of Roediger \& Hensler (2005) - dashed line. Our simulations: $R_{\mathrm{c}, \mathrm{ICM}}=3.4 \mathrm{kpc}-$ triangles; $6.7 \mathrm{kpc}$ - circles; $13.4 \mathrm{kpc}$ - filled circles; $26.8 \mathrm{kpc}$ - squares; $53.6 \mathrm{kpc}$ - diamonds. Solid line shows the prediction using Gunn \& Gott (1972) (Eq. (1)).

hydrodynamical effects such as formation of the bow shock in the ICM in front of the galaxy. It actually shields the galaxy, thereby reducing the efficiency of stripping.

\section{Discussion and conclusions}

The simulations performed in the present work allow us to estimate the consequences of ram pressure stripping in clusters better. If a galaxy like the Milky Way (our type LM) passes in face-on orientation through the central parts of a cluster similar to the Virgo, it loses about $30 \%$ of its interstellar gas. This constitutes a rather large amount of gas loss. According to Roediger \& Brüggen (2006), the efficiency of stripping only marginally depends on the orientation of the galaxy for inclinations lower than $\sim 60^{\circ}$. Therefore, we disregard this aspect in this discussion.

With this level of stripping, we may estimate the fraction of gas supplied to the ICM by stripping. About $2 \times 10^{9} M_{\odot}$ of gas is lost by a LM-type galaxy. A much less massive Lmtype galaxy is more severely stripped, thus roughly providing the same amount of gas. EM and Em-types are subject to an even more effective stripping, but their gas disks are less massive. Our estimate is that the average contribution per spiral galaxy, late or early type, with different orientations in a radial orbit, is $1-2 \times 10^{9} M_{\odot}$. This means that the total amount of hot gas in the central region of our standard cluster $\left(6 \times 10^{10} M_{\odot}\right)$ can be provided by $30-60$ stripping acts. The amount of stripping from past to present is influenced with the decreasing gas content of galaxies, which is an obvious consequence of gas consumption by star formation: gas-rich galaxies in the past can provide more gas to intergalactic space. In contrast, the growing ICM concentration in the center of the cluster leads to higher stripping efficiency now.

Note that only the contribution from galaxies on radial orbits is substantial. Galaxies on circular or elliptical orbits do not penetrate the dense parts of the ICM and thus are safe from being strongly stripped. Depending on the fraction of galaxies in radial orbits in the cluster, which unfortunately is not well known, the stripping could supply a significant amount of gas to the ICM. This may explain the high metallicity of the ICM gas.

Another consequence of our simulations is that we expect to find a significant amount of relatively cold gas in any cluster, 
as debris left over from recent stripping events. This gas is not yet mixed with the ICM, but forms large diffuse clouds as tails behind the galaxy keeping a fraction of its velocity. The mixing time should be comparable to the free-fall time, e.g. $\sim 1 \mathrm{Gyr}$. The density of gas in tails may be more than 10 times higher than the local hot ICM density, hence a tail could cause strong stripping for any galaxy that happens to cross it at a very different velocity. This can then provide a nice explanation of the puzzling strong stripping recently observed in many regions where the hot ICM gas is not sufficient to strip. For instance NGC 4522 is apparently stripped at a large distance from the cluster center (Crowl \& Kenney 2006), where the ICM density is not sufficient. A nice example of a cold $\mathrm{H}$ I tail almost $250 \mathrm{kpc}$ long provides the galaxy NGC 4254 in the Virgo cluster. This and similar tails may be the cause of ram pressure for other galaxies arriving at high speed. The surroundings of Virgo M 86 galaxy shows a complex structure in X-ray emission (Finoguenov et al. 2004), like a large plume extending in the northwest direction. However, the influence of such spatially narrow and small ICM peaks acting on a galaxy during a short time is estimated to be very slow. Also, ram-pressure stripping appears in small groups, where the X-ray gas is not detected (Rasmussen et al. 2006). As we have shown, the crucial effect of the ram pressure stripping is the pressure itself, so the hydrodynamical effects play only a minor role. Therefore, not only is the hot ICM able to strip the galactic ISM, but debris from tidal interactions or previous stripping do so, as well.

The main conclusion is that the stripping efficiency depends significantly on the duration of the ram pressure pulse, which in many cases means that the Gunn \& Gott (1972) prediction overestimates the amount of stripping. This is nicely shown by Boselli \& Gavazzi (2006, their Fig. 18), where they compare the restoring force in spiral galaxies in Coma, A1367 and Virgo clusters to the maximum ram pressure. It turns out that many galaxies with normal H I content should have been stripped if the ram pressure had acted long enough. Much more modest stripping is observed, showing that Gunn \& Gott (1972) prediction is an overestimate, since it neglects the role of reaccretion of the ISM. As encountered in Sect. 6.3, in early-type galaxies with high bulge-to-disk mass ratios, the maximum of the gravitational restoring force is more displaced from the disk plane than in late types. This means that the ram pressure can accelerate the disk gas more before it reaches the peak of the restoring force. In principle, this mechanism could allow stripping even when the Gunn \& Gott condition is not met. The right panel of Fig. 1 shows that, especially at larger disk radii, the maxima of the restoring force are well off the galaxy midplane. Our simulation results support this idea - in Fig. 21 galaxies crossing clusters with large ICM distributions show more stripping than corresponds to the Gunn \& Gott prediction.

\section{Summary}

Using the SPH/tree code GADGET (Springel et al. 2001) adapted for interactions of two gaseous phases, the ISM and the ICM, we have performed simulations of the ram pressure stripping of galaxies on completely radial orbits crossing galaxy clusters with realistic profiles of the ICM density.

We found different amounts of stripping (from complete to only marginal) in clusters with various ICM distributions.
However, when galaxies encounter the same amount of ICM on their orbits, the stripping results are found to be the same. Thus, for a given orbital type and cluster potential, the column density $\Sigma_{\text {ICM }}$ of the crossed ICM determines the stripping (see Fig. 15). We have proposed a simple analytic stripping criterion (Eq. (9)) taking the $\Sigma_{\text {ICM }}$ into account.

Since simulations with different levels of hydrodynamical treatment show similar results, we have suggested that the ram pressure is the dominant process in sweeping of galaxies. Although this also reflects the formation of a bow-shock structure in front of the galaxy due to its supersonic motion, we proposed the important role played by the ram pressure in the interactions of galaxies with debris structures in clusters.

We have indicated the limits of the Gunn \& Gott (1972) stripping formula, on one hand overestimating the amount of stripping since completely neglecting the time-dependence of the ISM-ICM interaction process, and on the other underestimating the stripping by omitting the accumulation of kinetic energy by the gas before it encounters the maximum of the restoring force.

Acknowledgements. The authors gratefully acknowledge support by the Institutional Research Plan AV0Z10030501 of the Academy of Sciences of the Czech Republic and by the project LC06014 Center for Theoretical Astrophysics. The majority of the simulations were carried out on the IBM Power 4 processors of the CNRS computing center at the IDRIS (Palaiseau, France). We would like to thank the anonymous referee for helping us to improve this paper considerably.

\section{References}

Abadi, M. G., Moore, B., \& Bower, R. G. 1999, MNRAS, 308, 947 Acreman, D. M., Stevens, I. R., Ponman, T. J., \& Sakelliou, I. 2003, MNRAS, 341,1333

Arnaboldi, M., Freeman, K. C., Okamura, S., et al. 2003, AJ, 125, 514

Barnes, J., \& Hut, P. 1986, Nature, 324, 446

Böhringer, H. 2004, in Recycling Intergalactic and Interstellar Matter, ed. P.-A.

Duc, J. Braine, \& E. Brinks, IAU Symp., 217, 92

Boselli, A., \& Gavazzi, G. 2006, PASP, 118, 517

Cavaliere, A., \& Fusco-Femiano, R. 1976, A\&A, 49, 137

Crowl, H. H., \& Kenney, J. D. P. 2006, ApJ, 649, L75

Domainko, W., Mair, M., Kapferer, W., et al. 2006, A\&A, 452, 795

Finoguenov, A., Pietsch, W., Aschenbach, B., \& Miniati, F. 2004, A\&A, 415, 415

Gingold, R. A., \& Monaghan, J. J. 1977, MNRAS, 181, 375

Gunn, J. E., \& Gott, J. R. I. 1972, ApJ, 176, 1

Hernquist, L. 1993, ApJS, 86, 389

Kundic, T., Spergel, D. N., \& Hernquist, L. 1993, BAAS, 25, 1428

Lucy, L. B. 1977, AJ, 82, 1013

Mihos, J. C. 2004, in Recycling Intergalactic and Interstellar Matter, ed. P.-A.

Duc, J. Braine, \& E. Brinks, IAU Symp., 217, 390

Moore, B., Katz, N., Lake, G., Dressler, A., \& Oemler, A. 1996, Nature, 379, 613

Murante, G., Arnaboldi, M., Gerhard, O., et al. 2004, ApJ, 607, L83

Oosterloo, T., \& van Gorkom, J. 2005, A\&A, 437, L19

Quilis, V., Moore, B., \& Bower, R. 2000, Science, 288, 1617

Rasmussen, J., Ponman, T. J., \& Mulchaey, J. S. 2006, MNRAS, 370, 453

Roediger, E., \& Brüggen, M. 2006, MNRAS, 369, 567

Roediger, E., \& Hensler, G. 2005, A\&A, 433, 875

Roediger, E., Brüggen, M., \& Hoeft, M. 2006, MNRAS, 371, 609

Schindler, S., Binggeli, B., \& Böhringer, H. 1999, A\&A, 343, 420

Schindler, S., Kapferer, W., Domainko, W., et al. 2005, A\&A, 435, L25

Schulz, S., \& Struck, C. 2001, MNRAS, 328, 185

Solanes, J. M., Giovanelli, R., \& Haynes, M. P. 1996, ApJ, 461, 609

Springel, V., Yoshida, N., \& White, S. D. M. 2001, New Astron., 6, 79

Vollmer, B., Cayatte, V., Balkowski, C., \& Duschl, W. J. 2001, ApJ, 561, 708

Vollmer, B., Soida, M., Otmianowska-Mazur, K., et al. 2006, A\&A, 453, 883 
P. Jáchym et al.: Gas stripping in galaxy clusters: a new SPH simulation approach, Online Material p 1

\section{Online Material}


P. Jáchym et al.: Gas stripping in galaxy clusters: a new SPH simulation approach, Online Material p 2

\section{Appendix A: More detailed simulations}

In Sect. 6.1, Figs. 9 and 10 show the distribution of the ICM density and relative velocity at five locations of the LM galaxy when it crosses the standard cluster. There, the ICM is represented with 120000 SPH particles. Figures A.1 and A.2 depict the same situation with $N_{\text {ICM }}=480000$. Now, ICM particles are smaller and the bow shock in front of the galaxy is more pronounced and ISM in the disk slightly better protected. However, as shown in Sect. 7, the stripping results are almost independent of the value of $N_{\text {ICM }}$. 
P. Jáchym et al.: Gas stripping in galaxy clusters: a new SPH simulation approach, Online Material p 3
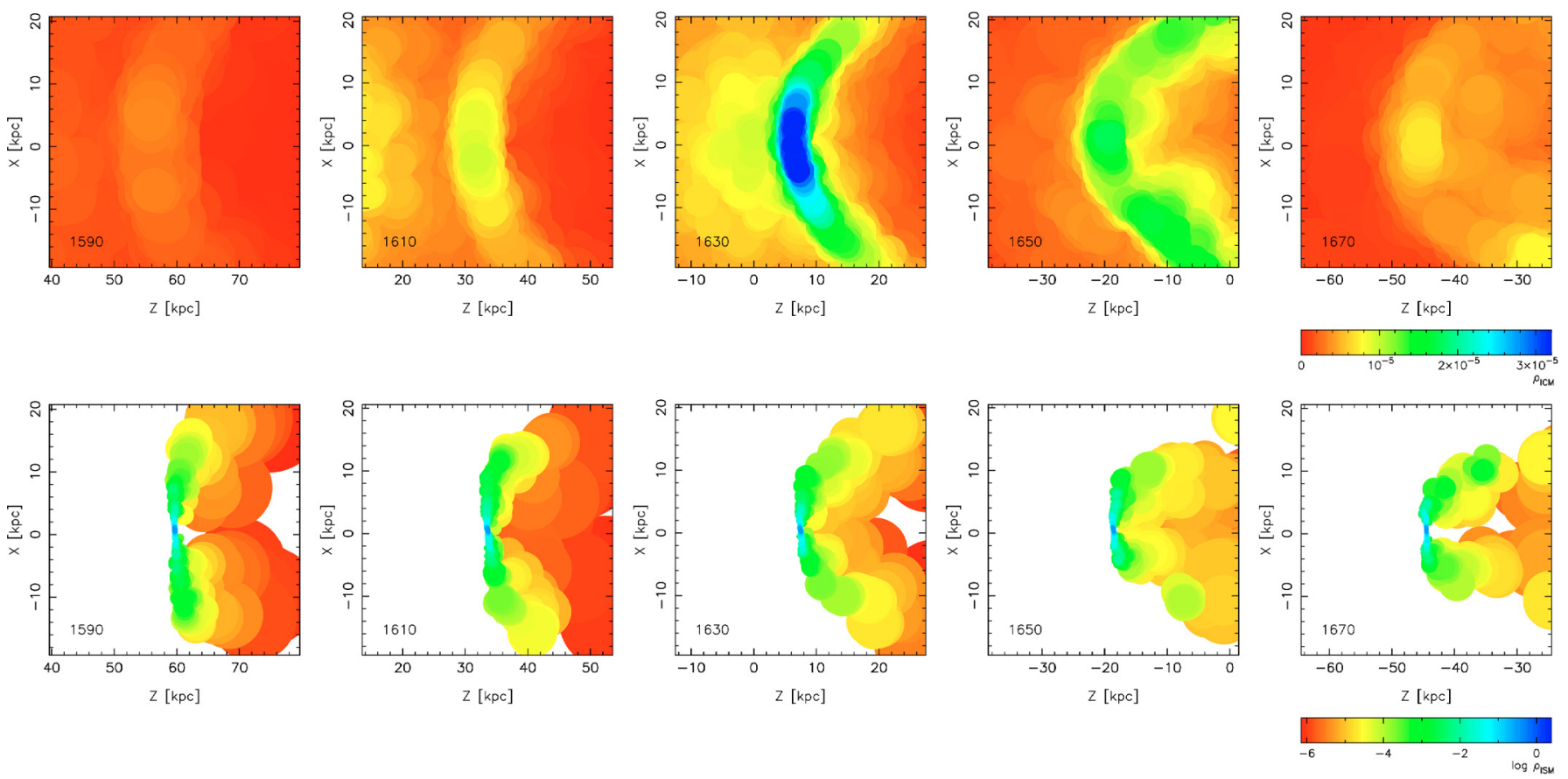

Fig. A.1. Density of the ICM in surroundings of the LM-type galaxy (top) and of the ISM (bottom). $N_{\mathrm{ICM}}=480000$. Particles are displayed as filled circles with radii equal to their SPH smoothing sizes. The ISM density scale is logarithmic. The time in Myr is given in the left lower corner of frames.
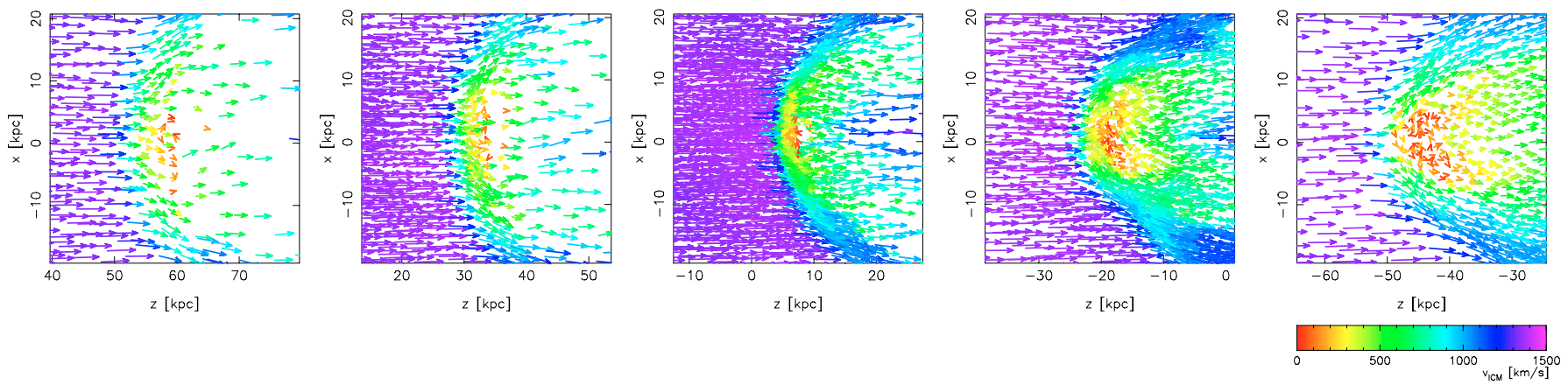

Fig. A.2. The flow of ICM particles around the LM-type galaxy in the case of $N_{\mathrm{ICM}}=480000$. 